Abstract-In this paper, a continuous-discrete time observer which simultaneously estimates the unmeasurable states and the uncertainties for the electro-hydraulic actuator (EHA) system is presented. The main feature of the proposed observer is the use of an intersample output predictor which allows the users to increase the frequency acquisition of the piston position sensor without affecting the convergence performance. The stability analysis of the proposed observer is proved using Lyapunov function adapted to hybrid systems. To show the efficiency of our proposed observer, numerical simulations and experimental validation involving a control application, which combines the designed observer and a PI controller for the purpose of piston position tracking problem, are presented.

Index Terms-Continuous-discrete time observers, disturbance observer (DOB), electro-hydraulic actuator (EHA), intersample output predictor, sampled data measurements.

\section{INTRODUCTION}

D UE TO a high power to weight ratio and their ability to generate high torques/forces outputs, electro-hydraulic actuator (EHA) systems are widely used in several industrial applications [1]-[5]. Despite this advantage, the EHA systems suffer from some drawbacks due principally to their structure. Indeed, the EHA systems are subject to various uncertainties such as model parametric variations [6], [7], highly nonlinear dynamic behavior [8], potential faults such as internal leakage [9], and hard damage affecting their functioning. In the last years, the increasing demand of high precision control for EHA systems renders the development of advance controls' methods necessary to meet the actual requirements in terms of tracking performance.

Despite their actual dominance, the traditional proportional integral derivative (PID) controllers are not robust enough to

Manuscript received March 31, 2015; revised August 5, 2015; accepted January 24, 2016. This work was supported by the Combustion Engine for Range-Extended Electric Vehicle (CEREEV) Project which is funded by the European Union's INTERREG IVA France-Manche-England Programme.

S. Ahmed Ali, A. Christen, and N. Langlois are with the Department of Electrical Engineering, IRSEEM/École Supérieure d'Ingénieurs en Génie Électrique (ESIGELEC), 76801 Rouen, France (e-mail: sofiane.ahmedali@esigelec.fr; Arnaud.Christen@esigelec.fr; nicolas.langlois@esigelec.fr).

$\mathrm{S}$. Begg is with the Department of Electrical Engineering, University of Brighton, Brighton, BN2 4GJ, U.K. (e-mail: S.M.Begg@ @righton.ac.uk).

Color versions of one or more of the figures in this paper are available online at http://ieeexplore.ieee.org.

Digital Object Identifier 10.1109/TIE.2016.2531022 counteract the effect of the uncertainties affecting the EHA systems. Therefore, the focus of the researchers has been shifted toward developing nonlinear closed-loop control methods in order to improve the tracking performance for the EHA systems. In the past decades, several nonlinear control techniques have been developed in the literature such as feedback linearization [7], [10] and sliding mode control [11]-[14]. In [6], a novel integration of adaptive control and integral robust feedback was proposed for hydraulic systems with considering all possible modeling uncertainties, and an excellent tracking performance was achieved, which is the first solution for theoretically asymptotic stability with unmatched disturbances for hydraulic systems; others nonlinear controllers such as robust/adaptive robust controllers [15]-[20], [37], [38] and backstepping control [21]-[24] were also proposed. These methods have already proved their efficiency to improve the tracking performance of the EHA systems facing modeling uncertainties, parametric variations, and external disturbances.

However, all aforementioned techniques are full-state feedback ones, i.e., the designed controllers assume that all states of the EHA systems are available for measurements. From practical of point of view, this assumption may not be realistic for some hydraulic systems. Indeed, for many hydraulics applications, only the position signal of the actuator is measured via sensor. The other states like velocity and hydraulic pressure are not measured because of the cost-reduction and the space limitation; therefore, states and disturbances observers have recently received in the literature more and more attention.

Several states and disturbances observers were developed by some researchers in the past decade. The idea behind developing these observers is to use the states and the disturbances estimation provided by these observers in order to synthesized an output-feedback controllers which compensate the internal and the external disturbances affecting the EHA systems. At this stage, we can distinguish between two main approaches in the literature. The first approach consists in developing only a state estimator (i.e., an observer) which estimates the unmeasurable state of the EHA systems. These observers ignore both the internal disturbances like parametric variations, modeling uncertainties, and the external disturbances such as the load and the friction torque affecting the hydraulic application. Those types of observers can be found in the work developed by the authors in [25]-[28]. The second approach developed by the authors in [29]-[31] assumes that the states of the EHA systems are measurable and synthesize a disturbance observer (DOB) 
which estimates the mechanical and the hydraulic disturbances affecting the system. These estimations are incorporated then in a nonlinear closed-loop controller which compensates the effect of the disturbance and improves the tracking performance of the desired position for the EHA systems.

Recently, the authors in [32] proposed a novel framework for the purpose of simultaneous estimation of the unmeasurable states and the unmodeled disturbances, and then resulting in an excellent output feedback nonlinear robust backstepping controller for hydraulic systems, by developing an extended state observer (ESO) [33] and robust backstepping design. In this work, the authors consider that the main uncertainties affecting the EHA systems come from the hydraulic part. Therefore, they synthesized an observer based on the well-known techniques of ESOs [33] which estimates the unmeasurable state and the hydraulic disturbances of the EHA systems. The proposed observer is also robust facing the mechanical disturbances generated by the load driven by the considered EHA system in this paper.

In the case of hydraulic applications, the main drawback of the designed observers [25]-[32] is that they assume that the measured variable is continuous. In practical situations, this measured variable which is given by the position sensor is sampled. In other words, the piston positions are available for the observer at only sampling times $t_{k}$ fixed by the sampling rate (i.e., the frequency acquisition) of the sensor. This frequency can affect the convergence of the proposed when it comes to the matter of implementation of the proposed observer on digital signal processors (DSPs).

Following the design in [32], the authors in [34] designed a sampled data observer which deals with the problem of discrete time-measurements for the EHA system. The proposed observer retains the same benefits which characterize the observer proposed in [32] in terms of simultaneous estimation of the unmeasurable states and the internal disturbances affecting the EHA system. The proposed observer involves in its structure an intersampled output predictor [35] which ensures continuous time estimation of the states and the exponential convergence of the observation errors. Moreover, the sampling period of the data acquisition of the observer can be augmented independently from the frequency acquisition of the sensor position without affecting the convergence of the observer. However, the designed observer in [34] suffers from two major drawback. The first one concerns the Lyapunov function provided to prove the exponential convergence of the proposed observer. Indeed, the authors in [34] demonstrated the exponential convergence of the observer only locally between two sampling periods. In addition, the performance of the proposed observer were validated only in simulations and no experimental validation of the observer is provided. Comparing to the work of the author in [34], two main contributions were provided. The first contribution consists in designing a novel Lyapunov function based on small gain arguments which guaranty a global exponential convergence of the proposed observer. In addition, the maximum sampling period $T_{\max }$ derived from this function is less restrictive comparing to the one derived in [34]. The second one is that experimental results performed on the experimental test rig of the Brighton University is provided

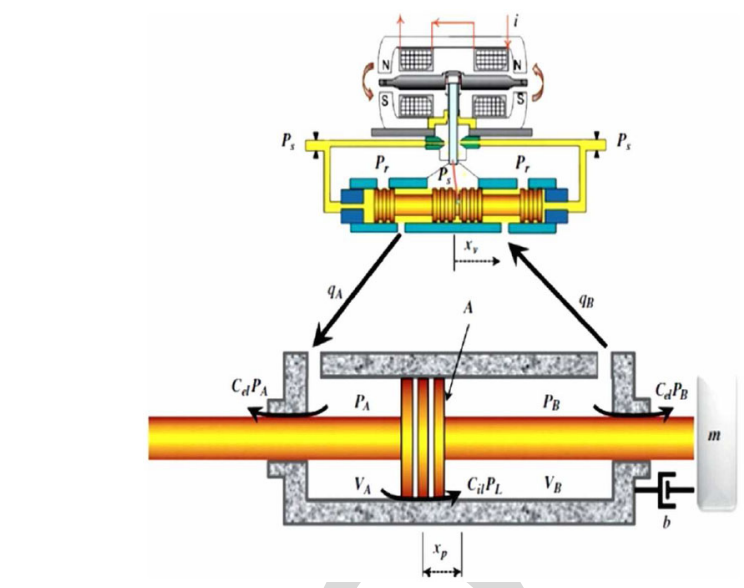

Fig. 1. Schematic of the EHA.

for this observer. This is in our acknowledged the first time that 141 such observers were designed and tested experimentally for the 142 EHA systems.

This paper is organized as follows. The EHA modeling 144 issues and the problem formulation are presented in Section II. 145 Section III presents the continuous-discrete time observer for 146 the EHA system. Numerical simulations and experimental val- 147 idation showing the effectiveness of our proposed observer are 148 presented in Section IV. Section V contains the conclusion and 149 the future works.

\section{EHA MODELING}

The schematic of the EHA studied in this paper is depicted in 152 Fig. 1 [26], [29]. The EHA system contains usually three parts, 153 namely the electrical, the mechanical, and the hydraulic part. 154 These parts represent an interconnected subsystem in such a 155 way that the dynamic of each subsystem influences the dynam- 156 ics of the others. The electrical part of the EHA system is a 157 servo-valve (top of Fig. 1) which controls the fluid dynamics 158 inside the chambers. The spool valve is driven by the electri- 159 cal input current $u$ of a torque motor. The displacement of the 160 spool valve $x_{v}$ together with the load pressure $P_{L}$ controls the 161 fluid dynamic inside two chambers A and B which constitute 162 the hydraulic part of the EHA system. The mechanical part of 163 the EHA system is a cylindrical piston which is modeled as 164 a classical mass-spring system. The position of the cylindrical 165 piston $x_{p}$ obeys to the fundamental principle of dynamics.

\section{A. State-Space Representation of the EHA}

Considering the following states variable: $x=168$ $\left[x_{1}, x_{2}, x_{3}\right]^{T}=\left[x_{p}, \dot{x_{p}}, P_{L}\right]^{T}$, the state-space representa- 169 tion of the EHA system can be written under the following 170 form [26], [29], [31]:

$$
\left\{\begin{array}{l}
\dot{x_{1}}=x_{2} \\
\dot{x_{2}}=-\frac{k}{m} x_{1}-\frac{b}{m} x_{2}+\frac{A_{p}}{m} x_{3} \\
\dot{x_{3}}=-\alpha x_{2}-\beta x_{3}+\gamma \sqrt{P_{s}-\operatorname{sign}(u) x_{3}} u
\end{array}\right.
$$


where $x_{p}$ is the piston position (m). $\dot{x}_{p}(\mathrm{~m} / \mathrm{s})$ is the piston velocity and $P_{L}(\mathrm{~Pa})$ is the pressure load inside the chambers of the hydraulic part. $k$ is the load spring constant $(\mathrm{N} / \mathrm{m}), b$ is the viscous damping coefficient $[\mathrm{N} /(\mathrm{m} / \mathrm{s})]$, and $A_{p}$ is the cylinder bore $\left(\mathrm{m}^{2}\right) . P_{s}$ is the supply pressure (Pa). $\alpha, \beta, \gamma$ are the hydraulic coefficients of the EHA model. These coefficients depend on the flow characteristics of the EHA system. For more details about the expression of the hydraulic coefficients $\alpha, \beta, \gamma$ and the modeling issues of the EHA system, the reader is referred to the work of the authors [26], [29] and their corresponding literature.

\section{B. Modeling Uncertainties and Disturbances Affecting the EHA System \\ Time-Varying}

In [29] and [31], the authors distinguished between two types of disturbances $d_{1}$ and $d_{2}$ which can affect the EHA system. The first one $d_{1}$ is the mechanical disturbance which is the result of lumping together the modeling parametric uncertainties, the load charge $F_{\text {Load }}$, and the friction force $F_{\text {friction }}$ acting on the mechanical part of the EHA system. As reported by the authors in [32], the second term $d_{2}$ does not hold the same significance as $d_{1}$. Indeed, $d_{2}$ represents the parametric deviation over the hydraulic coefficients $\alpha, \beta, \gamma$ and potential leakage affecting the hydraulic device of the EHA system. These parameters are also sensitive to temperature inside the EHA system. Taking into account these issues, the disturbed EHA model can be written as follows [29]:

$$
\left\{\begin{array}{l}
\dot{x_{1}}=x_{2} \\
\dot{x_{2}}=-\frac{k}{m} x_{1}-\frac{b}{m} x_{2}+\frac{A_{p}}{m} x_{3}-\frac{d_{1}}{m} \\
\dot{x_{3}}=-\alpha x_{2}-\beta x_{3}+\gamma \sqrt{P_{s}-\operatorname{sign}(u) x_{3}} u+d_{2}
\end{array}\right.
$$

where $d_{1}(t)$ and $d_{2}(t)$ are expressed as follows [31]:

$$
\begin{aligned}
& d_{1}(t)=-\triangle \frac{k}{m} x_{1}-\triangle \frac{b}{m} x_{2}-\triangle \frac{A_{p}}{m} x_{3}+F_{\text {Load }}+F_{\text {Friction }} \\
& d_{2}(t)=-\triangle \alpha x_{2}-\triangle \beta x_{3}+\triangle \gamma \sqrt{P_{s}-\operatorname{sign}(u) x_{3}} u
\end{aligned}
$$

The $\triangle$ symbolizes the considered parametric uncertainties affecting the mechanical and the hydraulic part of the EHA system. System (2) can be expressed under the following compact form:

$$
\left\{\begin{array}{l}
\dot{x}=A x+\varphi(x, u)+B_{d} d \\
y=C x=x_{1}
\end{array}\right.
$$

where $\boldsymbol{x} \in \mathbf{R}^{3}$ and $y \in \mathbf{R}$ represent, respectively, the state vector and the measured piston position $x_{1}=x_{p}$. The vector $\boldsymbol{u} \in$ $\mathbf{R}$ describes the set of admissible inputs. $d(\boldsymbol{t}) \in \mathbf{R}^{2}$ denotes the vector of the disturbances which affect the EHA. $B_{d}$ with dimensions $3 \times 2$. The matrices $A, B_{d}, C$, and vector $\phi(\boldsymbol{x}, \boldsymbol{u})$ have the following structure:

$$
A=\left(\begin{array}{ccc}
0 & 1 & 0 \\
0 & 0 & \frac{A_{p}}{m} \\
0 & 0 & 0
\end{array}\right)
$$

$$
\begin{aligned}
& B_{d}=\left(\begin{array}{cc}
0 & 0 \\
\frac{-1}{m} & 0 \\
0 & 1
\end{array}\right) \\
& C=\left(\begin{array}{lll}
1 & 0 & 0
\end{array}\right) \\
& \varphi(x, u)=\left(\begin{array}{c}
0 \\
-\frac{k}{m} x_{1}-\frac{b}{m} x_{2} \\
-\alpha x_{2}-\beta x_{3}+\gamma \sqrt{P_{s}-\operatorname{sign}(u) x_{3}} u
\end{array}\right) .
\end{aligned}
$$

\section{Problem Formulation}

For system (4), the piston position is available for measure- 210 ment only at each sampling times $t_{k}$ imposed by the frequency 211 acquisition (the sampling period) of the sensor manufacturer. In 212 this paper, we have to design a robust sampled data observer 213 which simultaneously estimates the unmeasurable states $x_{2}, 214$ $x_{3}$, and the hydraulic disturbance term $d_{2}$ of system (4). The 215 designed observer must deal with the sampling phenomenon of 216 the measured piston position $x_{p}$ and must be robust facing the 217 mechanical disturbance term $d_{1}(t)$. Under these considerations, 218 system (4) is rewritten as follows:

$$
\left\{\begin{array}{l}
\dot{x}=A x+\varphi(x, u)+B_{d} d \\
y\left(t_{k}\right)=C x\left(t_{k}\right)=x_{1}\left(t_{k}\right) .
\end{array}\right.
$$

System (5) combines a continuous dynamic behavior for the 220 states $x_{1}, x_{2}, x_{3}$ between two sampling times $\left[t_{k}, t_{k+1}\right]$ and an 221 updated step for the state $x_{1}$ which occurs at the sampling times 222 $t=t_{k}$.

\section{ill. Continuous-Discrete Time-Observer Design FOR THE EHA SYSTEM}

In this section, we design a continuous-discrete time 226 observer for the EHA system. Since $d_{2}$ is the main distur- 227 bance term, we use the well-known technique of the augmented 228 state system in order to estimate it. Following this, we add an 229 extended variable $x_{4}=d_{2}$ such as $\dot{x}_{4}=h(t)$ to system (5) so 230 that the augmented state system can be written as follows:

$$
\left\{\begin{array}{l}
\dot{\bar{x}}=\bar{A} \bar{x}+\overline{\varphi(\bar{x}, u)}+\delta(t) \\
y=\bar{C} \bar{x}=x_{1}
\end{array}\right.
$$

where $\bar{x}=\left[x_{1}, x_{2}, x_{3}, x_{4}\right]$ and

$$
\begin{aligned}
\bar{A} & =\left(\begin{array}{cccc}
0 & 1 & 0 & 0 \\
0 & 0 & \frac{A_{p}}{m} & 0 \\
0 & 0 & 0 & 1 \\
0 & 0 & 0 & 0
\end{array}\right) \\
\overline{\varphi(\bar{x}, u)} & =\left(\begin{array}{c}
0 \\
-\alpha x_{2}-\beta x_{3}+\gamma \sqrt{m} x_{1}-\frac{b}{m} x_{2} \\
0
\end{array}\right) \\
\delta(t) & =\left(\begin{array}{c}
0 \\
\frac{-d_{1}}{m} \\
0 \\
h
\end{array}\right) \\
\bar{C} & =\left(\begin{array}{ccc}
1 & 0 & 0
\end{array}\right) .
\end{aligned}
$$




\section{B. Observability Analysis}

From the structure of matrices $\bar{A}, \bar{C}$ in system (6), it can be easily checked that the pair $(\bar{A}, \bar{C})$ is observable. Hence, their exists two matrices $P, Q$ such that the following Lyapunov function is satisfied:

$$
P(\bar{A}-K \bar{C})+(\bar{A}-K \bar{C})^{T} P \leq-\mu \mathbb{I}_{n}
$$

The function $f$ is a saturation function which is introduced to guaranty that the estimated states $\hat{\bar{x}}$ remains inside the compact set so that the Lipschitz constant $\beta_{0}$ always exists. The $\triangle_{\theta}$ is a diagonal matrix $4 \times 4$ defined by

$$
\triangle_{\theta}=\left(\begin{array}{cccc}
1 & 0 & 0 & 0 \\
0 & \frac{1}{\theta} & 0 & 0 \\
0 & 0 & \frac{1}{\theta^{2}} & 0 \\
0 & 0 & 0 & \frac{1}{\theta^{3}}
\end{array}\right)
$$

where $\mu>0$ is a free-positive constant and $P$ is a symmetric 267 positive definite matrix.

Remark 3: Comparing to the work of the authors in [26], 269 [32], the novelty in the designed observer (8) is the introduc- 270 tion of the intersample output predictor term $w(t)$ [35] in the 271 correction term. The dynamic of this predictor is simply a copy 272 of the dynamics of system states equations. The role of the out- 273 put predictor term is to provide a continuous time prediction of 274 the output measured variable $y(t)$. Indeed, since the measured 275 output variable $y(t)$ is sampled, its values $y\left(t_{k}\right)$ are available 276 for the observer only at sampling times $t=t_{k}$. Comparing to 277 constant-gain zero-order-hold $(\mathrm{ZOH})$ approaches which main- 278 tain $y\left(t_{k}\right)$ constant between the sampling times, the output 279 predictor term $w(t)$ will provide a continuous time estimation 280 of $y(t)$ as it is the case in continuous time-observer design 281 framework.

Now, we are able to state the main results of this paper. 283

Theorem 1: Consider the EHA system (6), and suppose that 284 assumptions (1-2) holds, given a sampling period $T$, choose 285 $\sigma_{0}, \sigma_{1}, \sigma_{2}$ as in (17), define $\sigma_{3}=T e^{\sigma T} \frac{2 \sigma_{1}\left(\theta+\beta_{0}\right)}{\sigma_{0} \sqrt{\lambda_{\min }(P)}}$ then sys- 286 tem (8) is an exponential sampled data observer for system 287 (6) with the following properties: the vector of the observa- 288 tion error $\left\|\bar{e}_{\bar{x}}\right\|$ converges exponentially toward a ball whose 289 radius $R=\frac{2 \sigma_{2}}{\sigma_{0} \sqrt{\lambda_{\min }(P)}\left(1-\sigma_{3}\right)}$. Moreover, there exists a real 290 positive bounded $T_{\max }$ satisfying inequality (34), so that for all 291 $T \in\left(0, T_{\max }\right)$, the radius of the ball can be made as small as 292 desired by choosing large values of $\theta$ and $k_{i=1, \ldots, 4}$. 293

Proof 1: The proof of this theorem 1 is inspired from the 294 work of the authors in [35]. Let us now define the following 295 observer $e_{\bar{x}}$ and the output $e_{w}(t)$ errors as follows:

$$
\left\{\begin{array}{l}
e_{\bar{x}}(t)=\hat{\bar{x}}-\bar{x} \\
e_{w}(t)=w(t)-y(t)=w(t)-\bar{C} \bar{x}
\end{array}\right.
$$

Combining (6) and (8), we can easily check that for the EHA system (6), the following properties are satisfied: $\theta \triangle_{\theta}^{-1} \bar{A} \triangle_{\theta}=298$ $\theta \bar{A}$ and $\triangle_{\theta}^{-1} K \bar{C}=\triangle_{\theta}^{-1} K \bar{C} \triangle_{\theta}$. Introducing the well-known 299 change in coordinate in the high gain literature $\bar{e}_{\bar{x}}=\triangle_{\theta} e_{\bar{x}} \quad 300$ yields the following dynamics of the state and the output errors: 301

$$
\left\{\begin{aligned}
\dot{\bar{e}}_{\bar{x}}= & \theta(\bar{A}-K \bar{C}) \bar{e}_{\bar{x}}+\triangle_{\theta}(\overline{\varphi(f(\hat{\bar{x}}), u)}-\overline{\varphi(\bar{x}, u)}) \\
& +\theta K e_{w}-\triangle_{\theta} \delta(t) \\
\dot{e}_{w}= & \theta \bar{e}_{\bar{x} 2}+\left(\overline{\left.\varphi_{1}(f(\hat{\bar{x}})), u\right)}-\overline{\varphi_{1}(\bar{x}, u)}\right)
\end{aligned}\right.
$$

Let us now consider the following candidate Lyapunov 302 quadratic function $V=\bar{e}_{\bar{x}}^{T} P \bar{e}_{\bar{x}}$ :

$$
\begin{gathered}
\dot{V} \leq-\mu \theta\left\|\bar{e}_{\bar{x}}\right\|^{2}+2 \bar{e}_{\bar{x}}^{T} P \triangle_{\theta}(\overline{\varphi(f(\hat{\bar{x}}), u)}-\overline{\varphi(\bar{x}, u)}) \\
+2 \theta \bar{e}_{\bar{x}}^{T} P K e_{w}(t)-2 \bar{e}_{\bar{x}}^{T} P \triangle_{\theta} \delta
\end{gathered}
$$

Taking into account Assumptions (1-2) we have

$$
\begin{gathered}
\dot{V} \leq-\mu \theta\left\|\bar{e}_{\bar{x}}\right\|^{2}+4 \beta_{0} \lambda_{\max }(P)\left\|\bar{e}_{\bar{x}}\right\|^{2}+2 \theta\|P K\|\left\|\bar{e}_{\bar{x}}\right\|\left|e_{w}(t)\right| \\
+4 \lambda_{\max }(P)\left\|\bar{e}_{\bar{x}}\right\| \xi
\end{gathered}
$$

where $\xi=\sqrt{\mu_{1}^{2}+\mu_{2}^{2}}$. 
306

Using the well-known property

$$
\lambda_{\min }(P)\left\|\bar{e}_{\bar{x}}\right\|^{2} \leq V \leq \lambda_{\max }(P)\left\|\bar{e}_{\bar{x}}\right\|^{2}
$$

307 we derive

$$
\begin{aligned}
& \dot{V} \leq-\mu \theta \frac{V}{\lambda_{\max }(P)}+\frac{4 \beta_{0} \lambda_{\max }(P) V}{\lambda_{\min }(P)} \\
& +2 \theta\|P K\| \sqrt{\frac{V}{\lambda_{\min }(P)}}\left|e_{w}(t)\right|+4 \lambda_{\max }(P) \sqrt{\frac{V}{\lambda_{\min }(P)}} \xi .
\end{aligned}
$$

308 Now choosing the parameter $\theta$ such that $\theta>\theta_{0}$ with $\theta_{0}=$ $309 \sup \left\{1, \frac{8 \beta_{0} \lambda_{\max }^{2}(P)}{\mu \lambda_{\min }(P)}\right\}$, we have

$$
\begin{gathered}
\dot{V} \leq-\mu \theta \frac{V}{2 \lambda_{\max }(P)}+2 \theta\|P K\| \sqrt{\frac{V}{\lambda_{\min }(P)}}\left|e_{w}(t)\right| \\
+4 \lambda_{\max }(P) \sqrt{\frac{V}{\lambda_{\min }(P)}} \xi .
\end{gathered}
$$

310 Considering now the function $W=\sqrt{V}$, then we obtain

$$
\begin{gathered}
\dot{W} \leq-\mu \theta \frac{W}{4 \lambda_{\max }(P)}+\theta\|P K\| \frac{\left|e_{w}(t)\right|}{\sqrt{\lambda_{\min }(P)}} \\
+2 \frac{\lambda_{\max }(P)}{\sqrt{\lambda_{\min }(P)}} \xi .
\end{gathered}
$$

311 Let us set

$$
\left\{\begin{array}{l}
\sigma_{0}=\frac{\mu \theta}{4 \lambda_{\max }(P)} \\
\sigma_{1}=\frac{\theta\|P K\|}{\sqrt{\lambda_{\min }(P)}} \\
\sigma_{2}=2 \frac{\lambda_{\max }(P)}{\sqrt{\lambda_{\min }(P)}} .
\end{array}\right.
$$

312 Integrating (16), then

$$
\begin{gathered}
W(t) \leq e^{-\sigma_{0}\left(t-t_{0}\right)} W\left(t_{0}\right)+\sigma_{1} e^{-\sigma_{0} t} \int_{t_{0}}^{t} e^{\sigma_{0} s}\left|e_{w}(s)\right| d s \\
+\sigma_{2} e^{-\sigma_{0} t} \int_{t_{0}}^{t} e^{\sigma_{0} s}\|\xi(s)\| d s
\end{gathered}
$$

313 Multiplying both sides of (18) by $e^{\sigma t}$ and using the fact that $314 e^{-\left(\sigma_{0}-\sigma\right) t}<1$ we derive

$$
\begin{aligned}
e^{\sigma t} W(t) & \leq M\left(t_{0}\right)+\sigma_{1} e^{-\left(\sigma_{0}-\sigma\right) t} \int_{t_{0}}^{t} e^{\sigma_{0} s} \mid e_{w}(s \mid d s \\
& +\sigma_{2} e^{-\left(\sigma_{0}-\sigma\right) t} \int_{t_{0}}^{t} e^{\sigma_{0} s}\|\xi(s)\| d s
\end{aligned}
$$

315 where $M\left(t_{0}\right)=e^{\sigma_{0} t_{0}} W\left(t_{0}\right)$.

316 On the other hand, we have

$$
\begin{aligned}
e^{\sigma t} W(t) \leq & M\left(t_{0}\right)+\sigma_{1} e^{-\left(\sigma_{0}-\sigma\right) t} \int_{t_{0}}^{t} e^{\left(\sigma_{0}-\sigma\right) s} e^{\sigma s} \mid e_{w}(s \mid d s \\
& +\sigma_{2} e^{-\left(\sigma_{0}-\sigma\right) t} \int_{t_{0}}^{t} e^{\left(\sigma_{0}-\sigma\right) s} e^{\sigma s}\|\xi(s)\| d s
\end{aligned}
$$

317 or

$$
e^{\sigma t} W(t) \leq M\left(t_{0}\right)
$$

$$
\begin{aligned}
& +\sigma_{1} e^{-\left(\sigma_{0}-\sigma\right) t}\left(\int_{t_{0}}^{t} e^{\left(\sigma_{0}-\sigma\right) s} d s\right) \sup _{t_{0} \leq s \leq t}\left(e^{\sigma s}\left\|e_{w}(s)\right\|\right) \\
& +\sigma_{2} e^{-\left(\sigma_{0}-\sigma\right) t}\left(\int_{t_{0}}^{t} e^{\left(\sigma_{0}-\sigma\right) s} d s\right) \sup _{t_{0} \leq s \leq t}\left(e^{\sigma s}\|\xi(s)\|\right)
\end{aligned}
$$

which leads to

$$
\begin{aligned}
e^{\sigma t} W(t) \leq & M\left(t_{0}\right) \\
& +\frac{\sigma_{1}}{\sigma_{0}-\sigma} \sup _{t_{0} \leq s \leq t}\left(e^{\sigma s}\left|e_{w}(s)\right|\right) \\
& +\frac{\sigma_{2}}{\sigma_{0}-\sigma} \sup _{t_{0} \leq s \leq t}\left(e^{\sigma s}|| \xi(s) \|\right) .
\end{aligned}
$$

Now taking $0<\sigma<\sigma_{0} / 2$, we derive

$$
\begin{aligned}
\sup _{t_{0} \leq s \leq t}\left(e^{\sigma s} W(s)\right) \leq & M\left(t_{0}\right) \\
& +2 \frac{\sigma_{1}}{\sigma_{0}} \sup _{t_{0} \leq s \leq t}\left(e^{\sigma s}\left|e_{w}(s)\right|\right) \\
& +2 \frac{\sigma_{2}}{\sigma_{0}} \sup _{t_{0} \leq s \leq t}\left(e^{\sigma s}|| \xi(s) \|\right)
\end{aligned}
$$

and

$$
\begin{gathered}
W(t) \leq e^{-\sigma t} M\left(t_{0}\right)+2 \frac{\sigma_{1}}{\sigma_{0}} \sup _{t_{0} \leq s \leq t}\left(e^{-\sigma(t-s)}\left|e_{w}(s)\right|\right) \\
+2 \frac{\sigma_{2}}{\sigma_{0}} \sup _{t_{0} \leq s \leq t}\left(e^{-\sigma(t-s)}\|\xi(s)\|\right)
\end{gathered}
$$

which leads to

$$
\begin{aligned}
\left\|\bar{e}_{\bar{x}}\right\| & \leq e^{-\sigma t} \frac{M\left(t_{0}\right)}{\sqrt{\lambda_{\min }(P)}} \\
& +\frac{2 \sigma_{1}}{\sigma_{0} \sqrt{\lambda_{\min }(P)}} \sup _{t_{0} \leq s \leq t}\left(e^{-\sigma(t-s)} \mid e_{w}(s \mid)\right. \\
& +\frac{2 \sigma_{2}}{\sigma_{0} \sqrt{\lambda_{\min }(P)}} \sup _{t_{0} \leq s \leq t}\left(e^{-\sigma(t-s)}\|\xi(s)\|\right)
\end{aligned}
$$

and

$$
\begin{aligned}
\sup _{t_{0} \leq s \leq t}\left(e^{\sigma s}|| \bar{e}_{\bar{x}} \mid\right) & \leq \frac{M\left(t_{0}\right)}{\sqrt{\lambda_{\min }(P)}} \\
& +\frac{2 \sigma_{1}}{\sigma_{0} \sqrt{\lambda_{\min }(P)}} \sup _{t_{0} \leq s \leq t}\left(e^{\sigma s}\left|e_{w}(s)\right|\right) \\
& +\frac{2 \sigma_{2}}{\sigma_{0} \sqrt{\lambda_{\min }(P)}} \sup _{t_{0} \leq s \leq t}\left(e^{\sigma(s)}\|\xi(s)\|\right) .
\end{aligned}
$$

On the other hand, we have from (10) the following expression 323 of $\left|e_{w}(t)\right|$ :

$$
\left|e_{w}(t)\right|=\int_{t_{k}}^{t}\left|\theta \bar{e}_{\bar{x} 2}+\left(\overline{\varphi_{1}(f(\hat{\bar{x}}), u)}-\overline{\varphi_{1}(\bar{x}, u)}\right)\right| d s .
$$

Multiplying again both sides of (28) by $e^{\sigma t}$ and taking into 325 account assumptions $1-2$, we have

$$
e^{\sigma t}\left|e_{w}(t)\right| \leq e^{\sigma t}\left(\theta+\beta_{0}\right) \int_{t_{k}}^{t} e^{-\sigma s} e^{\sigma s}\left\|\bar{e}_{\bar{x}}(s)\right\| d s
$$

which leads to

$$
\begin{array}{r}
e^{\sigma t}\left|e_{w}(t)\right| \leq e^{\sigma t}\left(\theta+\beta_{0}\right)\left(\int_{t_{k}}^{t} e^{-\sigma s} d s\right) \\
\sup _{t_{k} \leq s \leq t}\left(e^{\sigma s}\left\|\bar{e}_{\bar{x}}(s)\right\|\right) d s
\end{array}
$$




$$
\begin{aligned}
& \sup _{t_{k} \leq s \leq t} e^{\sigma s}\left|e_{w}(s)\right| \leq T e^{\sigma T}\left(\theta+\beta_{0}\right) \\
& \sup _{t_{k} \leq s \leq t}\left(e^{\sigma s}\left\|\bar{e}_{\bar{x}}(s)\right\|\right) d s
\end{aligned}
$$

329 since $\sup _{t_{k} \leq s \leq t}\left(e^{\sigma s} \| \bar{e}_{\bar{x}}(s)\right) \leq \sup _{t_{0} \leq s \leq t}\left(e^{\sigma s} \| \bar{e}_{\bar{x}}(s)\right) \quad$ and taking into account that $t>t_{0}, t_{1}, \ldots, t_{k}$ we derive that

$$
\begin{aligned}
& \sup _{t_{0} \leq s \leq t} e^{\sigma s}\left|e_{w}(s)\right| \leq T e^{\sigma T}\left(\theta+\beta_{0}\right) \\
& \sup _{t_{0} \leq s \leq t}\left(e^{\sigma s}\left\|\bar{e}_{\bar{x}}(s)\right\|\right) d s
\end{aligned}
$$

Combining (32) with (27) we have

$$
\begin{aligned}
& \sup _{t_{0} \leq s \leq t}\left(e^{\sigma s}\left\|\bar{e}_{\bar{x}}\right\|\right) \leq \frac{M\left(t_{0}\right)}{\sqrt{\lambda_{\min }(P)}} \\
& \left.+T e^{\sigma T} \frac{2 \sigma_{1}}{\sigma_{0} \sqrt{\lambda_{\min }(P)}}\left(\theta+\beta_{0}\right) \sup _{t_{0} \leq s \leq t}\left(e^{\sigma s}\left\|\bar{e}_{\bar{x}}(s)\right\|\right) d s\right) \\
& +\frac{2 \sigma_{2}}{\sigma_{0} \sqrt{\lambda_{\min }(P)}} \sup _{t_{0} \leq s \leq t}\left(e^{\sigma(s)}\|\xi(s)\|\right)
\end{aligned}
$$

setting $\sigma_{3}=T e^{\sigma T} \frac{2 \sigma_{1}\left(\theta+\beta_{0}\right)}{\sigma_{0} \sqrt{\lambda_{\min }(P)}}$ then selecting $T_{\max }$ satisfying the following the small gain condition:

$$
T_{\max } e^{\sigma T_{\max }} \frac{2 \sigma_{1}\left(\theta+\beta_{0}\right)}{\sigma_{0} \sqrt{\lambda_{\min }(P)}}<1
$$

we have

$$
\begin{gathered}
\left\|\bar{e}_{\bar{x}}\right\| \leq e^{-\sigma t} \frac{M\left(t_{0}\right)}{\sqrt{\lambda_{\min }(P)}\left(1-\sigma_{3}\right)} \\
\left.+\frac{2 \sigma_{2}}{\sigma_{0} \sqrt{\lambda_{\min }(P)}\left(1-\sigma_{3}\right)} \sup _{t_{0} \leq s \leq t}\|\xi(s)\|\right) .
\end{gathered}
$$

This complete the proof of Theorem 1.

Remark 4: Contrary to ([34], (35) demonstrates the global exponential convergence of the vector of the observation error $\left\|\bar{e}_{\bar{x}}\right\|$ toward a ball whose radius depends on the magnitude of the disturbance vector $\boldsymbol{\xi}$. In addition, the maximum sampling period $T_{\max }$ derived in (34) is less restrictive comparing to the one derived in [34] which depends on the computation of a bounded positive function $\psi(t)$ (see (13) in [34]).

Remark 5: The radius of the ball $R$ is defined such that $R=$ $\frac{2 \sigma_{2}}{\sigma_{0} \sqrt{\lambda_{\min }(P)}\left(1-\sigma_{3}\right)}$. We also notice that in the case where there is no mechanical disturbances (i.e., $d_{1}=0$ ) and the hydraulic disturbances are constant or equal to 0 , we have an exponential convergence of the observation error $\left\|\bar{e}_{\bar{x}}\right\|$ toward 0 . Looking at the expression of the maximum sampling period $T_{\max }$ in (34), we can easily see that when $\sigma$ tends to zero, $T_{\max } \simeq \frac{1}{\theta}$. Hence, augmenting $\theta$ will diminish the value of $T_{\max }$. On the other hand, large values of parameter $\theta$ will contribute to reduce the radius $R$ and hence to improve the performance of our observer. However, it is well known that the high gain observers literature, augmenting the values of $\theta$ will lead to the undesirable peaking phenomenon which consists in an impulsive behavior of the states estimation trajectory around initial conditions.
TABLE I

Numerical Parameter Values for the Eha System

\begin{tabular}{|c|c|}
\hline Parameters & Value \\
\hline$m$ & 0.5 \\
\hline$b$ & 0 \\
\hline$k$ & $5.651110 \times 10^{5}$ \\
\hline$A_{p}$ & $5.058 \times 10^{-4}$ \\
\hline$k_{v}$ & $1.333 \times 10^{-5}$ \\
\hline$\alpha$ & $3.257 \times 10^{10}$ \\
\hline$\beta$ & 2.146 \\
\hline$\gamma$ & $7.169 \times 10^{9}$ \\
\hline$P_{s}$ & $2.1 \times 10^{7}$ \\
\hline
\end{tabular}

TABLE II

$\mathrm{T} 2: 1$

Parameters of THE Hybrid OBSERVER

$\mathrm{T} 2: 2$

\begin{tabular}{|c||c|c|c|}
\hline \multirow{4}{*}{ Parameter } & $\theta$ & $K=\left(\begin{array}{c}K_{1} \\
K_{2} \\
K_{3} \\
K_{4}\end{array}\right)$ & $T_{s}$ \\
\hline
\end{tabular}

IV. Simulations and Experimental Results

A. Numerical Simulation of the Hybrid Observer Coupled 358 With PI Controller for the EHA System Subject to 359 Mechanical and Hydraulic Disturbances

The performance of the proposed observer will be evaluated 361 first under MATLAB/Simulink Software. For the purpose of 362 comparison, the numerical simulations were performed on the 363 EHA system validated experimentally by the authors in [26] 364 and [29]. The model parameters' values are shown in Table I. 365

In this numerical simulations, we will demonstrate the 366 effectiveness of our proposed observer in terms of states/ 367 disturbances estimation and positioning control. In [29], the 368 authors considered a sinusoidal reference position signal $x_{1 d}=369$ $0.008 \sin (2 \pi t)$. For the purpose of tracking $x_{1 d}$, a PI controller 370 was employed and combined with the proposed observer (8) so 371 that the novel PI control law $u$ is expressed as follows:

$$
u=K_{p}\left(w(t)-x_{1 d}\right)+K_{i} \int\left(w(t)-x_{1 d}\right)
$$

where $x_{1}=x_{p}$ is the piston position and $K_{p}=3.18 \times 373$ $10^{-2}, K_{i}=100$ are the PI gains. The PI controller gains were 374 tuned in order to track. The numerical simulations were per- 375 formed using the Runge-Kutta solver with a fixed step size 376 $T_{\text {sim }}=10^{-4}$ s. The parameters of the hybrid observer are sum- 377 marized in Table II where $T_{s}$ is the sampling period of our 378 proposed hybrid (continuous-discrete time) observer.

The values of the observer parameters used in this simulation 380 are $\theta=1000, K=(10,35,49426,23724)$ and $T_{s}=1 \mathrm{~ms} . \quad 381$

The evaluation of our observer is performed under the con- 382 sideration that both mechanical and hydraulic disturbances 383 affect the considered EHA system in this paper. For the 384 mechanical disturbance term $d_{1}$, we have taken the same one 385 considered by the authors [29]. To show the robustness of our 386 observer facing the mechanical disturbances, we considered 387 it in the simulation not from the beginning but at $t=10 \mathrm{~s} .388$ Hence, the term $d_{1}$ in the disturbed model of the EHA in (2) 389 

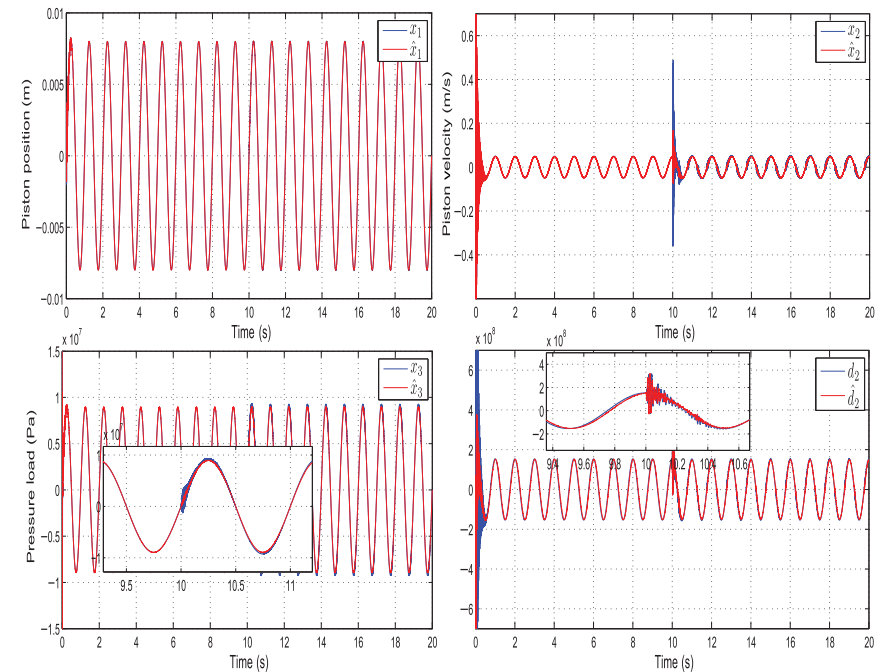

F2:1 Fig. 2. Estimation of $x_{1}, x_{2}, x_{3}, d_{2}$ for $\theta=1000$ and $T_{s}=1 \mathrm{~ms}$ with F2:2 mechanical and hydraulic disturbances. is expressed as follows:

$$
d_{1}(t)= \begin{cases}0, & \text { if } t<10 \mathrm{~s} \\ 294 \sin \left(62.83 x_{1}\right)+20 \operatorname{sign}\left(x_{2}\right), & \text { if } t \geq 10 \mathrm{~s}\end{cases}
$$

We also assume in this simulation that $10 \%$ additive parametric variation affects the hydraulic coefficients $\gamma$; hence (see Section II), the hydraulic disturbance term $d_{2}$ takes the following form:

$$
d_{2}(t)=10 \% \sqrt{P_{s}-\operatorname{sign}(u) x_{3}} u .
$$

From Fig. 2, we can see that the tracking performance of the reference $x_{1 d}$ even in the presence of the mechanical disturbance at $t=10 \mathrm{~s}$ is achieved correctly by the PI controller (36). The robustness of the PI controller facing the mechanical disturbance can be also seen in Fig. 2 where we can see that this disturbance has no effect on the tracking performance of the motion reference trajectory $x_{1 d}$. For the estimation of the piston velocity $x_{2}$, the pressure load $x_{3}$, and the hydraulic disturbance term $d_{2}$, we can see the effect of the mechanical disturbance (see Fig. 2 top right, bottom left, and right) which consists in a deviation of the states estimation trajectory occurring at $t=10 \mathrm{~s}$. Meanwhile, this deviation is quickly rejected by the observer, thanks to the large value of parameter $\theta$ taken in this simulation. As mentioned in Remark 5, large values of parameter $\theta$ will lead to a better rejection of the mechanical and the hydraulic disturbance term, however, this will amplify the peaking phenomenon which consists in an impulsive behavior of the trajectory of the states estimation at the beginning of the simulation (see Fig. 2).

\section{B. Performance Comparison With the Observer Designed in [26] and [32]}

To show the performance of our proposed observer, we have performed a comparison with the observers designed in [26] and [32]. Indeed, the observers [26], [32] have the same high gain like observer structure as the one considered in the design
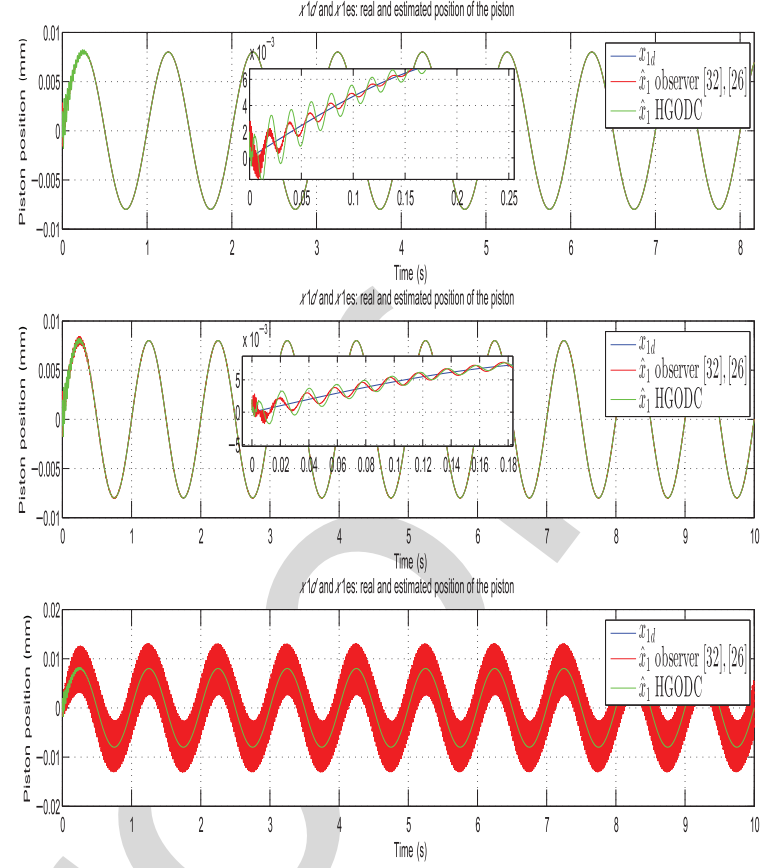

Fig. 3. Comparison of position tracking performance between our F3:1 observer [high gain observer discrete-continuous (HGODC)] $\left(T_{s}=\mathrm{F} 3: 2\right.$ $1 \mathrm{~ms}$ ) and observers [26], [32] (top: $T_{s}=0.1 \mathrm{~ms}$; middle: $T_{s}=0.5 \mathrm{~ms} ; \mathrm{F} 3: 3$ bottom: $T_{s}=1 \mathrm{~ms}$ ).

$\mathrm{F} 3: 4$

of our observer. By taking into account the sampling effect in 420 the structure of these two observers, a continuous-discrete time 421 version of the observers designed in [26] and [32] can be written 422 as follows:

423

$$
\dot{\overline{\hat{x}}}=\bar{A} \hat{\bar{x}}+\overline{\varphi(f(\hat{\bar{x}}), u)}-H\left(\bar{C} \hat{\bar{x}}(t)-y\left(t_{k}\right)\right) .
$$

We notice that in the case of our observer $H=\theta \triangle_{\theta}^{-1} K$. The 424 structure of (37) uses the sampled data $y\left(t_{k}\right)$ in the correction 425 term since that continuous measured variable $y(t)$ is available 426 only at sampled instants $t=t_{k}$. The simulations presented in 427 Fig. 3 show the performance of observer (8) and observer (37) 428 in terms of position tracking performances. For our proposed 429 observer (named HGODC), we have fixed the value of $T_{s}$ to 430 $1 \mathrm{~ms}$. For observer (37), three values were taken $\left(T_{s}=0.1,431\right.$ 0.5, and $1 \mathrm{~ms}$ ). Looking at Fig. 3 (top), we can see that even 432 if observer (37) performs better in the transitory regime, our 433 observer has quite the same performance. Recalling that in this 434 case, $T_{s}=0.1 \mathrm{~ms}$ for observer (37) which is the same sampling 435 period as the one of the solver, we can say that our observer 436 recovers the performances of continuous time observers. When 437 augmenting the sampling period of observer (37) to $0.5 \mathrm{~ms}, 438$ we can see that for observer (37), the performance degrades. 439 Finally, when the two observers have the same sampling peri- 440 ods ( $\left.T_{s}=1 \mathrm{~ms}\right)$, observer (37) diverges and the PID controller, 441 which is based on the estimation provided by observer (37), 442 fails to track the desired trajectory $x_{1 d}$.

\section{Experimental Validation}

To illustrate the performance of our proposed observer, an 445 experimental test rig platform has been set up and photographed 446 


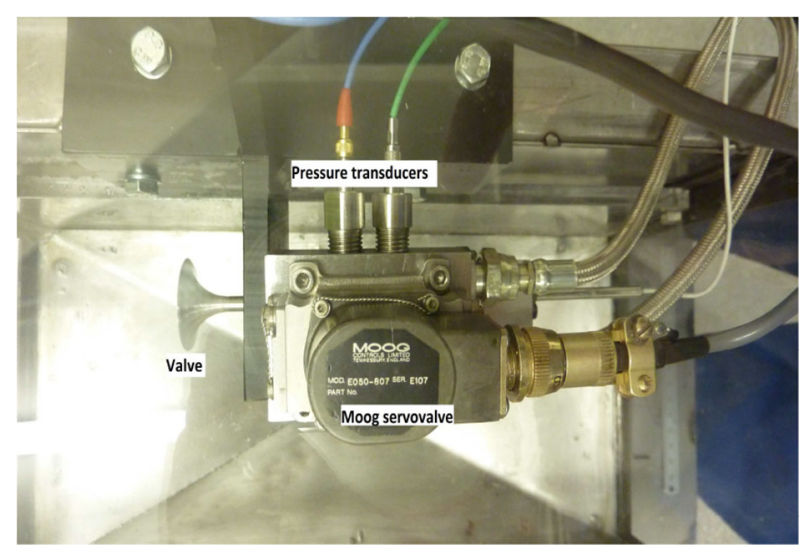

F4:1 Fig. 4. Moog servo-valve and the EHA actuator assembly.

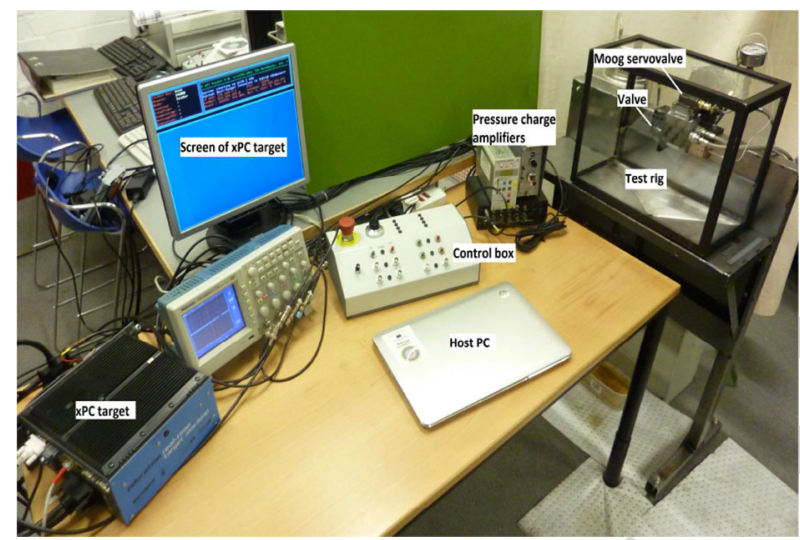

F5:1 Fig. 5. Control system of the experimental test rig of the EHA system.

in Figs. 4 and 5. The test rig was constructed in the Brighton University to investigate the performance of the EHA assembly and the control parameters influencing the motion of the poppet valve. The test rig comprised of three main subsystems: a hydraulic oil pressure supply; a hydraulic valve actuation assembly; and the servo-valve control signal and valve position interface.

Hydraulic oil from a large tank was supplied to a smaller reservoir coupled to a high-pressure pump and accumulator. An electromagnetic pressure-limit switch was used to regulate the supply of high-pressure oil to the hydraulic valve actuation assembly via an oil filter. The supply pressure was regulated to 70 bar \pm 2 bar by a pressure-limit switch.

The actuator body housed a double-acting hydraulic piston, oil-sealing end plates, and the high-pressure oil supply and return feed lines. A continuous-proportional (four-way) directional servo-valve (Moog series 31) was used to control the flow rate of hydraulic oil to the hydraulic piston by means of a proportional electromagnetic servo control signal. The interchangeable poppet valve head was attached to one end of the hydraulic piston and a linear variable differential transducer (LVDT) was mounted to the opposite end to record the change in valve position. The calibration factor for the amplified output of the LVDT sensor (Lord MicroStrain) was $2.97 \mathrm{~mm} / \mathrm{V} \pm 0.005 \mathrm{~mm} / \mathrm{V}$. Two piezoelectric gauge pressure
TABLE III

EHA Parameter Values for the Experimental Test Rig

\begin{tabular}{|c|c|}
\hline Parameters & Value \\
\hline$m$ & 0.05 \\
\hline$k$ & 2000 \\
\hline$b$ & 0.1398 \\
\hline$A_{p}$ & 0.0614 \\
\hline$k_{v}$ & 0.02 \\
\hline$\alpha$ & 28.2226 \\
\hline$\beta$ & 0.0063 \\
\hline$\gamma$ & 0.0029 \\
\hline$P_{s}$ & $7 \times 10^{6}$ \\
\hline
\end{tabular}

transducers (Kistler type 6125 transducer and type 5011 ampli- 472 fier) were used to measure the instantaneous and difference in 473 oil pressures in the supply and return chambers either sides of 474 the hydraulic piston. The pressure transducer was calibrated to 475 $20 \mathrm{bar} / \mathrm{V}$. The full-scale error in the transducer was \pm 3 bar. The 476 value of the oil pressure at the instant of initial piston motion 477 was used as the gauge reference pressure.

The control system for the electro-hydraulic valve system 479 was based on a real-time simulation and testing platform 480 (hardware in the loop, HIL); MathWorks MATLAB Simulink 481 and XPC Target application and a real-time target machine 482 (Speedgoat $\mathrm{GmbH}$ ). Positional feedback of the valve was deter- 483 mined from the LVDT sensor output. The actuation of the 484 directional servo-valve was achieved using a current driver sig- 485 nal rated to $\pm 50 \mathrm{~mA}$. The displacement of the poppet valve is 486 comprised between [20-32] mm. Based on the physical param- 487 eters of the experimental test rig [36], the nominal values of the 488 EHA model parameters were identified and listed in Table III. 489

In the following experiments, the parameters' values of 490 the hybrid observer for this experiment are $\theta=500, K=491$ $(2.8,2.87,1.0423,0.1710)$, and $T_{s}=1 \mathrm{~ms}$.

\section{PID Control Design for the Experimental Test Rig}

In order to track the motion reference $x_{1 d}$, the following 494 PID control law $u$ with a velocity feedforward action was 495 implemented

$$
\begin{gathered}
u=K_{p}\left(x_{1 d}-w(t)\right)+K_{i} \int\left(x_{1 d}-w(t)\right) \\
+K_{d} \frac{d}{d t}\left(x_{1 d}-w(t)\right)+K_{f} \dot{x}_{1 d}
\end{gathered}
$$

where $K_{p}=0.54, K_{i}=1.93, K_{d}=0.04, K_{f}=1$. As it was 497 the case in the simulation section, the implemented control law 498 $u$ contains the output prediction term $w(t)$. We mention that for 499 this experimental validation, we used the same Runge-Kutta 500 solver with the same fixed step size $T_{\text {sim }}=10^{-4}$ as in the 501 numerical simulations section. The experimental validation was 502 conducted with a sampling period $T_{s}=1 \mathrm{~ms}$ which is 10 times 503 bigger than the fixed step size of the solver.

\section{E. Experimental Performances of the Hybrid Observer 505 Without Disturbance

In this section, we investigate the performance of the hybrid 507 observer for state estimation and piston position tracking 508 

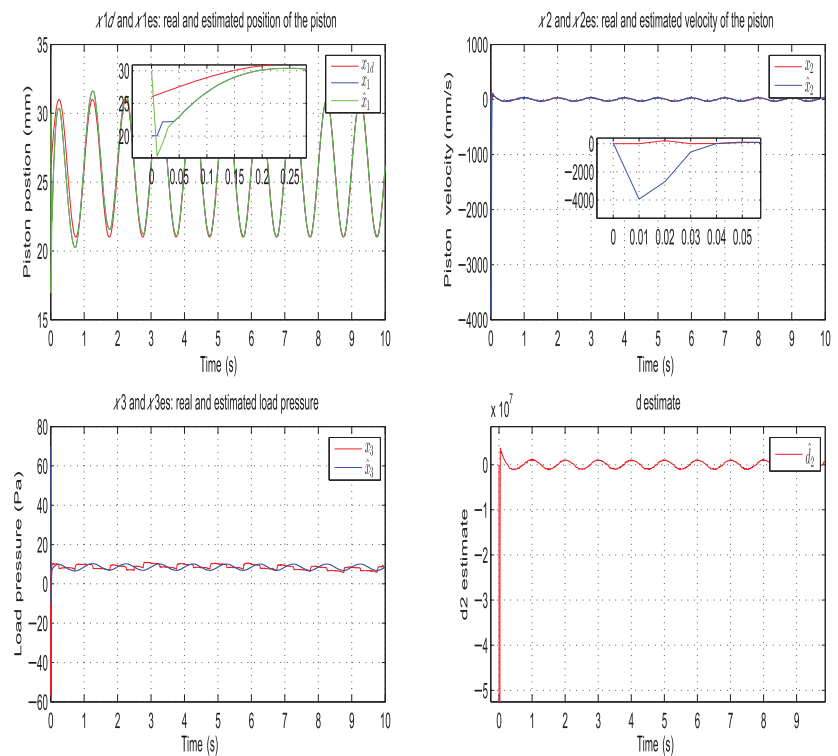

F6:1 Fig. 6. Estimation and tracking performance of $x_{1}, x_{2}, x_{3}, d_{2}$ for F6:2 $\theta=500, T_{s}=1 \mathrm{~ms}$ for EHA system without disturbances.

motion trajectory $x_{1 d}=26+5 \sin (2 \pi t)$. Since the considered EHA system does not drive any mechanical load, we have theoretically $d_{1} \simeq=0$. We also mention that we have used the same nominal values of the EHA system when implementing the hybrid observer.

In Fig. 6 (top left), we show the performance of the hybrid observer in terms of tracking performances and state estimation of the piston position $x_{1}$. We can see in Fig. 6 (top left) that both the tracking performance and the state estimation are achieved correctly by the hybrid observer. For the state estimation of the piston position $x_{1}$, the convergence of the hybrid observer is achieved with small convergence rate [less than $0.05 \mathrm{~s}$ when looking to the zoom of Fig. 6 (top left)]. We can see also that the tracking performance of the motion reference $x_{1 d}$ by the PI controller, which uses the output predictor $w(t)$, is also achieved correctly.

Fig. 6 (top right) shows the state estimation of the piston velocity $x_{2}$. We can see in Fig. 6 (top right) that our hybrid observer provides a very good estimation of the real piston velocity $x_{2}$. A quick look to Fig. 6 (top right) shows that the effect noise, which comes from the numerical differentiation used to obtain the real piston, has been attenuated by our hybrid observer.

In Fig. 6 (bottom left), we present the estimation results of the hydraulic pressure state $x_{3}$ by our proposed observer. First, we can observe from Fig. 6 (bottom left) that our observer provides a good estimation of the hydraulic pressure state $x_{3}$ despite the variations in the hydraulic parameters and the hydraulic disturbance which affects the functioning of the EHA system. The effects of these disturbances can be viewed. In Fig. 6 (bottom right) where we can see that even if there is no mechanical load driven by the EHA system, the estimated disturbance term $\hat{d}_{2}$ is not equal to 0 . Indeed, the difficulty of capturing the hydraulic parameters $(\alpha, \beta, \gamma)$ and the internal leakage occurring on the EHA system generates automatically the disturbance term $d_{2}$. For the reader, we mention that it was
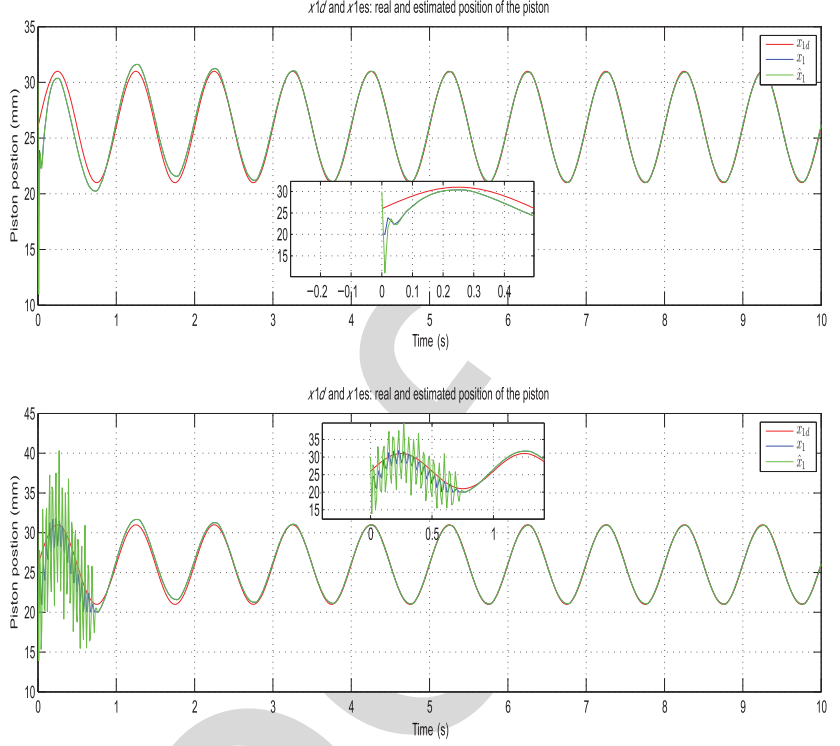

Fig. 7. Estimation and tracking performance of $x_{1}$ for $\theta=500$. (Top) F7:1 $T_{s}=2 \mathrm{~ms}$. (Bottom) $T_{s}=3 \mathrm{~ms}$.

very difficult for us to plot in Fig. 6 (bottom right) the real 545 hydraulic disturbance term $d_{2}$ for the reasons explained above. 546 Finally, we can observe in Fig. 6 (bottom left) that there is 547 small phase lag between the real and the estimated hydraulic 548 pressure $x_{3}$. This observation is quite interesting because of the 549 discrepancies between the numerical simulations and the exper- 550 imental validation of our observer. This discrepancies come 551 from the difficulty of capturing exactly the hydraulic parame- 552 ters of the EHA system and the fact that the dynamic of the 553 electrical part of the EHA system has been neglected in the 554 EHA model. In addition, it appears that the PID control is not 555 able to compensate it. Taking into account that the kistler pres- 556 sure transducers give a relative and not an absolute pressures 557 values in each chamber of the hydraulic actuator, we can say 558 that the estimated hydraulic pressures provided by our observer 559 are good.

\section{F. Effect of the Sampling Period on the Performance of 561 the Hybrid Observer

To compute the maximum allowable sampling period $T_{\max } 563$ of the hybrid observer, we can proceed following two pos- 564 sible manners. The first one is to compute $T_{\max }$ analytically 565 using the expression in (34); however, this will necessitate to 566 know the constant $\beta_{0}$ which is practically very difficult to deter- 567 mine. The second one is to start with a sampling period $T_{s}$ and 568 increasing it until the observer diverges. We proceed follow- 569 ing the second manner. In Fig. 7, we present the experimental 570 results of the estimated piston position $x_{1}$ and the tracking per- 571 formance of the piston position reference $x_{1 d}$. We mention that 572 we did not report the experimental results concerning the esti- 573 mations of the piston velocity $x_{2}$, the hydraulic pressure $x_{3}, 574$ and the hydraulic disturbances $d_{2}$. The reason is that they are 575 characterized by the same dynamic behavior as the results pre- 576 sented in Fig. 7. When increasing $T_{s}$ to $2 \mathrm{~ms}$, we can observe 577 from the top of Fig. 7 that the estimated piston position and 578 

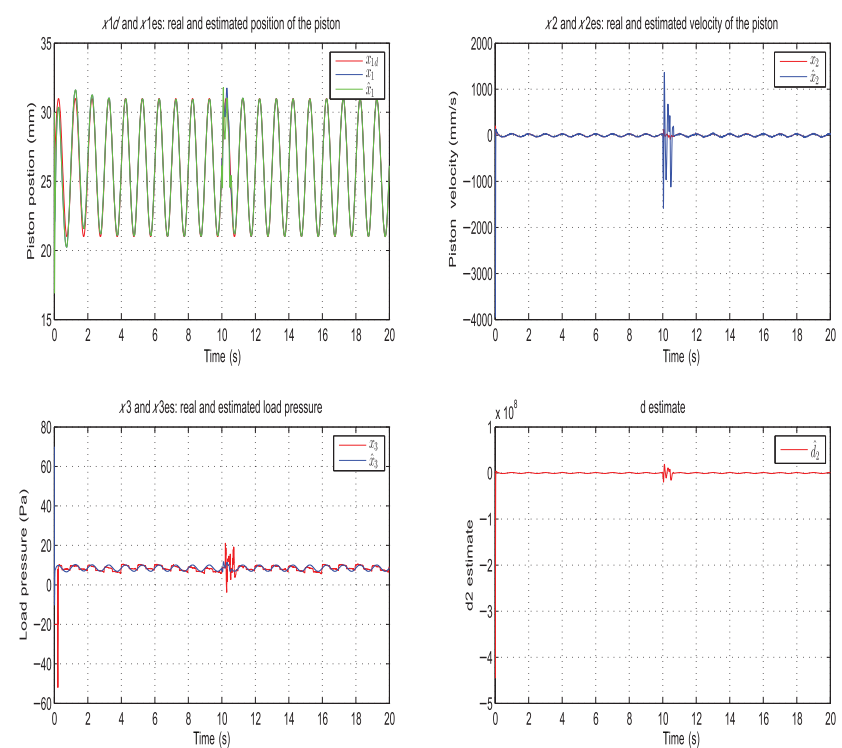

F8:1 Fig. 8. Estimation and tracking performance of $x_{1}, x_{2}, x_{3}, d_{2}$ for F8:2 $\theta=500, T_{s}=1 \mathrm{~ms}$ for EHA system with disturbances.

the tracking performance are quite the same as it is the case of $T_{s}=1 \mathrm{~ms}$. The difference concerns the convergence speed which is slower in the case of $T_{s}=1 \mathrm{~ms}$. When increasing $T_{s}$ to $3 \mathrm{~ms}$, we can observe that the performances of the hybrid observer are affected only in the transitory regime (see bottom of Fig. 7). Indeed, the oscillations observed in the bottom of Fig. 7 are due to the increase in the sampling period $T_{\max }$ to $3 \mathrm{~ms}$ which clearly affects the transitory regime for our hybrid observer. In the permanent regime, the hybrid observer which provides the output predictor term $w(t)$ for the PID controller performs well in the case of estimation and the tracking performance. From this, we can deduce that in the case of this experimental results, $T_{\max } \simeq 2 \mathrm{~ms}$.

\section{G. Experimental Performances of the Hybrid Observer With Disturbance}

To investigate the performance of our observer in the presence of disturbance, an additional disturbance term $d_{3}=2 x_{1 d}$ is inserted in the control input at $t=10 \mathrm{~s}$; meanwhile, the new control input sent to the control board is $u 1=u+2 x_{1 d}$, where $u$ is the previous control calculated by the PID controller. According to the structure of the model of the EHA system, this disturbance will be added to the previously hydraulic disturbance term $d_{2}$ and will change the dynamic of the states $\left(x_{1}, x_{2}, x_{3}, x_{4}\right)$ of the EHA system. We can see from Fig. 8 that both tracking performances and states estimation are achieved correctly by our observer. At $t=10 \mathrm{~s}$, we can see the influence of the disturbances on the performances of our observer. Despite its occurrence, we can clearly say that: first, the PID controller is robust facing this disturbance; since that the PID control law $u$ uses the predictor term $w(t)$ provided by our observer, this will demonstrate the easiness of the incorporation of our observer in a control scheme; second, our observer succeeds to estimate the states and the disturbances affecting the EHA system after $(t=10 \mathrm{~s})$.

\section{CONCLUSION AND FUTURE WORK}

In this paper, a continuous-discrete time observer is designed 614 for the EHAs system subject to discrete time measurement and 615 mechanical and hydraulic disturbances. The exponential con- 616 vergence of the proposed observer is proven using a classical 617 quadratic Lyapunov function based on small gain arguments. 618 The proposed observer is combined with PID controller for the 619 purpose of tracking motion reference trajectory of the piston 620 position for the EHA system. The simulation results and the 621 experimental validation of our proposed observer demonstrate 622 its efficiency in terms of tracking performance and distur- 623 bance estimation. In our future works, we plan to synthesize an 624 output feedback controllers based on the designed continuous- 625 discrete time observer in this paper. The resulting controllers 626 will improve the positioning control for the EHAs system.

\section{REFERENCES}

[1] J. Yao, Z. Jiao, and S. Han, "Friction compensation for low velocity 629 control of hydraulic flight motion simulator: A simple adaptive robust 630 approach," Chin. J. Aeronaut., vol. 26, no. 3, pp. 841-822, Jun. 2013.631

[2] J. Yao, Z. Jiao, B. Yao, Y. Shang, and W. Dong, "Nonlinear adaptive 632 robust control of electrohydraulic load simulator," Chin. J. Aeronaut., 633 vol. 25, no. 5, pp. 766-775, Oct. 2012.

[3] W. Sun, H. Gao, and O. Kaynak, "Adaptive backstepping control for 635 active suspension systems with hard constraints," IEEE/ASME Trans. 636 Mechatronics, vol. 18, no. 3, pp. 1072-1079, Jun. 2013.

[4] Y. Pi and X. Wang, "Observer-based cascade control of a 6-DOF parallel 638 hydraulic manipulator in joint space coordinate," Mechatronics, vol. 20, 639 no. 6, pp. 648-655, Sep. 2010.

[5] W. Sun, Y. Zhao, J. Li, L. Zhang, and H. Gao, "Active suspension control 641 with frequency band constraints and actuator input delay," IEEE Trans. 642 Ind. Electron., vol. 59, no. 1, pp. 530-537, Jan. 2012.

[6] J. Yao, Z. Jiao, D. Ma, and L. Yan, "High-accurac of hydraulic rotary actuators with modeling uncertainties," IEEE/ASME 645 Trans. Mechatronics, vol. 19, no. 2, pp. 633-641, Apr. 2014.

[7] H. A. Mintsa, R. Venugopal, J.-P. Kenne, and C. Belleau, "Feedback 647 linearization-based position control of an electrohydraulic servo system 648 with supply pressure uncertainty," IEEE Trans. Control Syst. Technol., 649 vol. 20, no. 4, pp. 1092-1099, Jul. 2012.

[8] H. E. Merritt, Hydraulic Control Systems. Hoboken, NJ, USA: Wiley, 1967.

[9] J. Yao, G. Yang, and D. Ma, "Internal leakage fault detection and tolerant control of single-rod hydraulic actuators," Math. Prob. Eng., vol. 2014.

[10] J.-H. Kwon, T.-H. Kim, J.-S. Jang, and I.-S. Lee, "Feedback linearization control of a hydraulic servo system," in Proc. SICE-ICASE Int. Joint Conf., 2006, pp. 455-460.

[11] H.-M. Chen, J.-C. Renn, and J.-P. Su, "Sliding mode control with varying 658 boundary layers for an electro-hydraulic position servo system," Int. J. 659 Adv. Manuf. Technol., vol. 26, no. 1, pp. 117-123, 2005.

12. A Ghazy "Variable structure control for electrohydraulic position 661 servo system," in Proc. IEEE 27th Annu. Conf. Ind. Electron. Soc., 2001, 662 pp. 2194-2198.

[13] C. Guan and S. Pan, "Adaptive sliding mode control of electro-hydraulic system with nonlinear unknown parameters," Control Eng. Pract., vol. 16, no. 11, pp. 1275-1284, Nov. 2008.

[14] Y. Lin, Y. Shi, and R. Burton, "Modeling and robust discrete-time sliding 667 mode control design for a fluid power electro-hydraulic actuator (EHA) 668 system," IEEE/ASME Trans. Mechatronics, vol. 18, no. 1, pp. 1-10, Feb. 669 2013

[15] A. G. Loukianov, J. Rivera, Y. Orlov, and E. Teraoka, "Robust trajectory tracking for an electrohydraulic actuator," IEEE Trans. Ind. Electron., vol. 56, no. 9, pp. 3523-3531, Sep. 2009.

[16] B. Yao, F. Bu, J. Reedy, and G. T. C. Chiu "Adaptive robust motion 674 control of single-rod hydraulic actuators: Theory and experiments," 675 IEEE/ASME Trans. Mechatronics, vol. 5, no. 1, pp. 79-91, Mar. 2000.676

[17] B. Yao, F. Bu, and G. T. C. Chiu, "Nonlinear adaptive robust con- 677 trol of electro-hydraulic systems driven by double-rod actuators," Int. J. 678 Control., vol. 74, no. 8, pp. 761-775, Aug. 2001. 
[18] G. Cheng and P. Shuangxia, "Nonlinear adaptive robust control of single-rod electro-hydraulic actuator with unknown nonlinear parameters," IEEE Trans. Control Syst. Technol., vol. 16, no. 3, pp. 434-445, May 2008.

[19] C. Wang, Z. Jiao, S. Wu, and Y. Shang, "Nonlinear adaptive torque control of electro-hydraulic load system with external active motion disturbance," Mechatronics, vol. 24, no. 1, pp. 32-40, Feb. 2014.

Y. Cungui and Q. Xianwei, "Simplified adaptive robust motion control with varying boundary discontinuous projection of hydraulic actuator," Math. Prob. Eng., vol. 2014.

[21] A. Alleyne and R. Liu, "A simplified approach to force control for electrohydraulic systems," Control Eng. Pract., vol. 8, no. 12, pp. 1347-1356. Dec. 2000.

[22] C. Kaddissi, J.-P. Kenne, and M. Saad, "Identification and real-time control of an electrohydraulic servo system based on nonlinear backstepping," IEEE/ASME Trans. Mechatronics, vol. 12, no. 1, pp. 12-22, Feb. 2007.

[23] C. Kaddissi, J.-P. Kenne, and M. Saad, "Indirect adaptive control of an electrohydraulic servo system based on nonlinear backstepping," IEEE/ASME Trans. Mechatronics, vol. 16, no. 6, pp. 1171-1177, Dec. 2011.

[24] K. K. Ahn, D. N. C. Nam, and M. Jin, "Adaptive backstepping control of an electrohydraulic actuator," IEEE/ASME Trans. Mechatronics, vol. 19, no. 3, pp. 987-995, Jun. 2014.

[25] P. Nakkarat and S. Kuntanapreeda, "Observer-based backstepping force control of an electrohydraulic actuator," Control Eng. Pract., vol. 17, no. 8, pp. 895-902, 2009.

[26] K. Wonhee, W. Daehee, S. Donghoon, and C. Chung, "Output feedback nonlinear control for electro-hydraulic systems," Mechatronics, vol. 22, no. 6, pp. 766-777, Sep. 2012.

[27] X. Wang, X. Sun, S. Li, and H. Ye, "Output feedback domination approach for finite-time force control of an electrohydraulic," IET Control Theory, vol. 6, no. 7, pp. 921-934, 2011.

[28] H. Khan, S. C. Abou, and N. Sepehri, "Nonlinear observer-based fault detection technique for electro-hydraulic servo-positioning systems," Mechatronics, vol. 15, no. 9, pp. 1037-1059, 2005.

[29] K. Wonhee, W. Daehee, S. Donghoon, and C. Chung, "Disturbanceobserver-based position tracking controller in the presence of biased sinusoidal disturbance for electrohydraulic actuators," IEEE Trans. Control Syst. Technol., vol. 21, no. 6, pp. 2290-2298, Nov. 2013.

[30] C. S. Kim and C. O. Lee, "Speed control of an overcentered variable displacement hydraulic motor with a load torque observer," Control Eng. Pract., vol. 4, no. 11, pp. 1563-1570, 1996.

[31] W. Daehee, K. Wonhee, S. Donghoon, and C. Chung, "High-gain disturbance observer-based backstepping control with output tracking error constraint for electro-hydraulic systems," IEEE Trans. Control Syst. Technol., vol. 23, no. 6, pp. 758-795, Mar. 2014.

[32] J. Yao, Z. Jiao, and D. Ma, "Extended-state-observer-based output feedback nonlinear robust control of hydraulic systems with backstepping," IEEE Trans. Ind. Electron., vol. 61, no. 11, pp. 6285-6293, Nov. 2014.

[33] J. Yao, Z. Jiao, and D. Ma, "Adaptive robust control of dc motors with extended state observer," IEEE Trans. Ind. Electron., vol. 61, no. 7, pp. 3630-3637, Jul. 2014.

[34] S. Ahmed Ali, "Sampled data observer based inter-sample output predictor for electro-hydraulic actuators," ISA Trans., vol. 58, pp. 421-433, 2015.

[35] I. Karafyllis and C. Kravaris, "From continuous-time design to sampleddata design of observers," IEEE Trans. Autom. Control, vol. 54, no. 9 , pp. 2169-2174, Sep. 2009.

[36] A. Karakayis and S. Begg, "Investigation of control system strategies for hydraulic valve actuation in an IC engine Adil Karakayis," M.S. thesis, Dept. Autom. Eng., Univ. Brighton School Comput., Eng. Math., Div. Eng. Product Des., Brighton, U.K., 2014.

[37] W. Sun, Z. Zhao, and H. Gao, "Saturated adaptive robust control for active suspension systems," IEEE Trans. Ind. Electron., vol. 60, no. 9, pp. 38893896, Sep. 2013.

[38] W. Sun, H. Gao, and B. Yao, "Adaptive robust vibration control of full-car active suspensions with electrohydraulic actuators," IEEE Trans. Control Syst. Technol., vol. 21, no. 6, pp. 2417-2422, Nov. 2013.

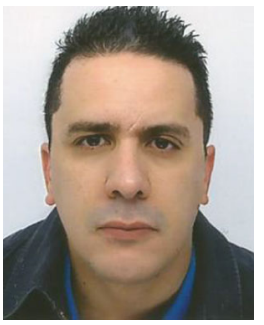

Sofiane Ahmed Ali was born in Algiers, 749 Algeria, in 1977. He received the B.Sc. degree 750 in electrical engineering from the University 751 of Technology Houari Boumediene, Algiers, 752 Algeria, in 2001, and the M.Sc. and Ph.D. 753 degrees in electrical and computer engineer- 754 ing from the University of Le Havre, Le Havre, 755 France, in 2004 and 2008, respectively. $\quad 756$

In 2008, he was appointed as a Research 757 and Development Engineer with Renault. Since 758 2010, he has been a Teaching and Research 759 Assistant Professor with the École Supérieure d'Ingénieurs en Génie Électrique (ESIGELEC), Rouen, France. His research interests include sliding mode control, nonlinear observers and fault-tolerant control, and diagnosis in the field of mechatronics devices.

\section{0}

761 $762 \bigcirc$

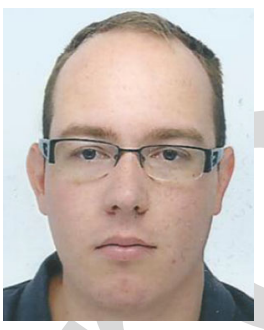

Arnaud Christen was born in France, in 764 1991. He received the Baccalaureate degree 765 in science (with honors) in 2009, before fol- 766 lowing a two-year preparation in mathemat- 767 ics and physics for entrance to the French 768 Engineering Schools. He received the dual M.S. 769 degree in control theory (electrical engineer- 770 ing) and mechatronics from École Supérieure 771 d'Ingénieurs en Génie Électrique (ESIGELEC), Rouen, France, and the University of Rouen, Rouen, France, in 2015.
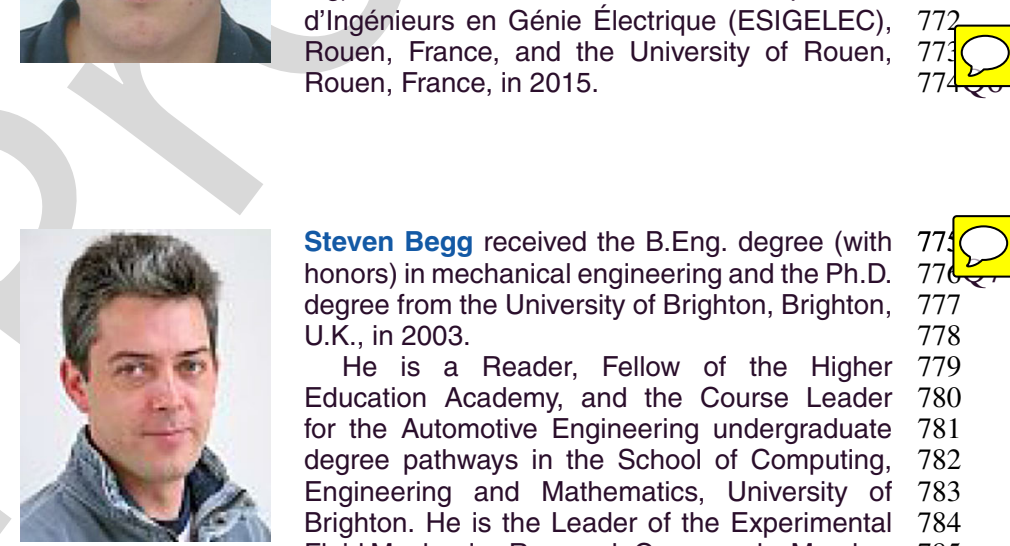

Steven Begg received the B.Eng. degree (with honors) in mechanical engineering and the Ph.D. degree from the University of Brighton, Brighton, U.K., in 2003.

$\mathrm{He}$ is a Reader, Fellow of the Higher Education Academy, and the Course Leader for the Automotive Engineering undergraduate degree pathways in the School of Computing Engineering and Mathematics, University of Brighton. He is the Leader of the Experimental Fluid Mechanics Research Group and a Member of the Advanced Engineering Centre at Brighton, U.K. He has led 786 applied research programmes (EPSRC, DfT, DTI, TSB, and EU) as 787 well as industrial consultancy projects, in the fields of automotive engi- 788 neering, fluid mechanics, and optical diagnostic techniques, for over 21789 years.

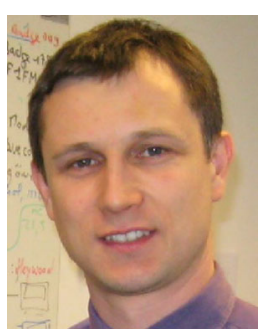

Nicolas Langlois received the Ph.D. and HDR 791 (habilitation to supervise research) degrees in 792 automatic control and signal processing from the 793 University of Rouen, Rouen, France, in 2001 and 794 2008, respectively.

In 2000, he joined th $Q$ aduate School 796 of Electrical Engineering, LELEC, Rouen, 797 France. $\mathrm{He}$ is currently the Head-in-Charge 798 of skills acquisition through research of 799 ESIGELEC, where he teaches courses on 800 control systems and digital signal processing. 801 $\mathrm{He}$ has also the Head of the "Automatic Control and Systems" research 802 team at the research institute IRSEEM since 2008. His research 803 interests include fault-tolerant control.

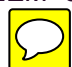




\section{QUERIES}

Q1: Please provide expansion for "PI."

Q2: Please provide expansion for "IRSEEM."

Q3: As per IEEE style, vectors have been changed to boldface italic. Please check whether all the occurrences are identified correctly and specify the missed out occurrences.

Q4: Please provide complete details of Refs. [9] and [20].

Q5: Please provide the location for Renault in the biography section of the author Sofiane Ahmed Ali.

Q6: Please provide the institution name and location for the Baccalaureate degree of the author Arnaud Christen.

Q7: Please spell out the term "ESIGELEC."

Q8: Please provide field of study for the Ph.D. degree of author "Steven Begg."

Q9: Please expand "IRSEEM" in the biography section. 


\title{
Continuous-Discrete Time-Observer Design for State and Disturbance Estimation of Electro-Hydraulic Actuator Systems
}

\author{
Sofiane Ahmed Ali, Arnaud Christen, Steven Begg, and Nicolas Langlois
}

\begin{abstract}
In this paper, a continuous-discrete time observer which simultaneously estimates the unmeasurable states and the uncertainties for the electro-hydraulic actuator (EHA) system is presented. The main feature of the proposed observer is the use of an intersample output predictor which allows the users to increase the frequency acquisition of the piston position sensor without affecting the convergence performance. The stability analysis of the proposed observer is proved using Lyapunov function adapted to hybrid systems. To show the efficiency of our proposed observer, numerical simulations and experimental validation involving a control application, which combines the designed observer and a PI controller for the purpose of piston position tracking problem, are presented.
\end{abstract}

Index Terms-Continuous-discrete time observers, disturbance observer (DOB), electro-hydraulic actuator (EHA), intersample output predictor, sampled data measurements.

\section{INTRODUCTION}

D UE TO a high power to weight ratio and their ability to generate high torques/forces outputs, electro-hydraulic actuator (EHA) systems are widely used in several industrial applications [1]-[5]. Despite this advantage, the EHA systems suffer from some drawbacks due principally to their structure. Indeed, the EHA systems are subject to various uncertainties such as model parametric variations [6], [7], highly nonlinear dynamic behavior [8], potential faults such as internal leakage [9], and hard damage affecting their functioning. In the last years, the increasing demand of high precision control for EHA systems renders the development of advance controls' methods necessary to meet the actual requirements in terms of tracking performance.

Despite their actual dominance, the traditional proportional integral derivative (PID) controllers are not robust enough to

Manuscript received March 31, 2015; revised August 5, 2015; accepted January 24, 2016. This work was supported by the Combustion Engine for Range-Extended Electric Vehicle (CEREEV) Project which is funded by the European Union's INTERREG IVA France-Manche-England Programme.

S. Ahmed Ali, A. Christen, and N. Langlois are with the Department of Electrical Engineering, IRSEEM/École Supérieure d'Ingénieurs en Génie Électrique (ESIGELEC), 76801 Rouen, France (e-mail: sofiane.ahmedali@esigelec.fr; Arnaud.Christen@esigelec.fr; nicolas.langlois@esigelec.fr).

$\mathrm{S}$. Begg is with the Department of Electrical Engineering, University of Brighton, Brighton, BN2 4GJ, U.K. (e-mail: S.M.Begg@ @ brighton.ac.uk).

Color versions of one or more of the figures in this paper are available online at http://ieeexplore.ieee.org.

Digital Object Identifier 10.1109/TIE.2016.2531022 counteract the effect of the uncertainties affecting the EHA systems. Therefore, the focus of the researchers has been shifted toward developing nonlinear closed-loop control methods in order to improve the tracking performance for the EHA systems. In the past decades, several nonlinear control techniques have been developed in the literature such as feedback linearization [7], [10] and sliding mode control [11]-[14]. In [6], a novel integration of adaptive control and integral robust feedback was proposed for hydraulic systems with considering all possible modeling uncertainties, and an excellent tracking performance was achieved, which is the first solution for theoretically asymptotic stability with unmatched disturbances for hydraulic systems; others nonlinear controllers such as robust/adaptive robust controllers [15]-[20], [37], [38] and backstepping control [21]-[24] were also proposed. These methods have already proved their efficiency to improve the tracking performance of the EHA systems facing modeling uncertainties, parametric variations, and external disturbances.

However, all aforementioned techniques are full-state feedback ones, i.e., the designed controllers assume that all states of the EHA systems are available for measurements. From practical of point of view, this assumption may not be realistic for some hydraulic systems. Indeed, for many hydraulics applications, only the position signal of the actuator is measured via sensor. The other states like velocity and hydraulic pressure are not measured because of the cost-reduction and the space limitation; therefore, states and disturbances observers have recently received in the literature more and more attention.

Several states and disturbances observers were developed by some researchers in the past decade. The idea behind developing these observers is to use the states and the disturbances estimation provided by these observers in order to synthesized an output-feedback controllers which compensate the internal and the external disturbances affecting the EHA systems. At this stage, we can distinguish between two main approaches in the literature. The first approach consists in developing only a state estimator (i.e., an observer) which estimates the unmeasurable state of the EHA systems. These observers ignore both the internal disturbances like parametric variations, modeling uncertainties, and the external disturbances such as the load and the friction torque affecting the hydraulic application. Those types of observers can be found in the work developed by the authors in [25]-[28]. The second approach developed by the authors in [29]-[31] assumes that the states of the EHA systems are measurable and synthesize a disturbance observer (DOB) 
which estimates the mechanical and the hydraulic disturbances affecting the system. These estimations are incorporated then in a nonlinear closed-loop controller which compensates the effect of the disturbance and improves the tracking performance of the desired position for the EHA systems.

Recently, the authors in [32] proposed a novel framework for the purpose of simultaneous estimation of the unmeasurable states and the unmodeled disturbances, and then resulting in an excellent output feedback nonlinear robust backstepping controller for hydraulic systems, by developing an extended state observer (ESO) [33] and robust backstepping design. In this work, the authors consider that the main uncertainties affecting the EHA systems come from the hydraulic part. Therefore, they synthesized an observer based on the well-known techniques of ESOs [33] which estimates the unmeasurable state and the hydraulic disturbances of the EHA systems. The proposed observer is also robust facing the mechanical disturbances generated by the load driven by the considered EHA system in this paper.

In the case of hydraulic applications, the main drawback of the designed observers [25]-[32] is that they assume that the measured variable is continuous. In practical situations, this measured variable which is given by the position sensor is sampled. In other words, the piston positions are available for the observer at only sampling times $t_{k}$ fixed by the sampling rate (i.e., the frequency acquisition) of the sensor. This frequency can affect the convergence of the proposed when it comes to the matter of implementation of the proposed observer on digital signal processors (DSPs).

Following the design in [32], the authors in [34] designed a sampled data observer which deals with the problem of discrete time-measurements for the EHA system. The proposed observer retains the same benefits which characterize the observer proposed in [32] in terms of simultaneous estimation of the unmeasurable states and the internal disturbances affecting the EHA system. The proposed observer involves in its structure an intersampled output predictor [35] which ensures continuous time estimation of the states and the exponential convergence of the observation errors. Moreover, the sampling period of the data acquisition of the observer can be augmented independently from the frequency acquisition of the sensor position without affecting the convergence of the observer. However, the designed observer in [34] suffers from two major drawback. The first one concerns the Lyapunov function provided to prove the exponential convergence of the proposed observer. Indeed, the authors in [34] demonstrated the exponential convergence of the observer only locally between two sampling periods. In addition, the performance of the proposed observer were validated only in simulations and no experimental validation of the observer is provided. Comparing to the work of the author in [34], two main contributions were provided. The first contribution consists in designing a novel Lyapunov function based on small gain arguments which guaranty a global exponential convergence of the proposed observer. In addition, the maximum sampling period $T_{\max }$ derived from this function is less restrictive comparing to the one derived in [34]. The second one is that experimental results performed on the experimental test rig of the Brighton University is provided

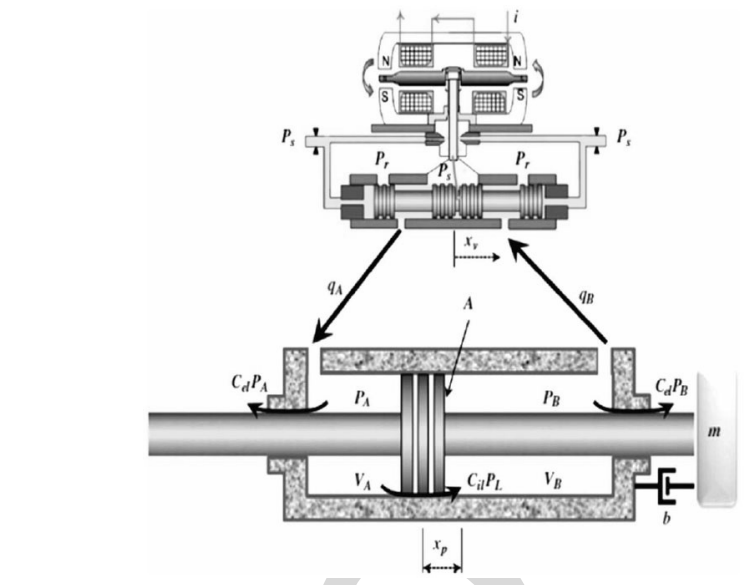

Fig. 1. Schematic of the EHA.

for this observer. This is in our acknowledged the first time that 141 such observers were designed and tested experimentally for the 142 EHA systems.

This paper is organized as follows. The EHA modeling 144 issues and the problem formulation are presented in Section II. 145 Section III presents the continuous-discrete time observer for 146 the EHA system. Numerical simulations and experimental val- 147 idation showing the effectiveness of our proposed observer are 148 presented in Section IV. Section V contains the conclusion and 149 the future works.

\section{EHA MODELING}

The schematic of the EHA studied in this paper is depicted in 152 Fig. 1 [26], [29]. The EHA system contains usually three parts, 153 namely the electrical, the mechanical, and the hydraulic part. 154 These parts represent an interconnected subsystem in such a 155 way that the dynamic of each subsystem influences the dynam- 156 ics of the others. The electrical part of the EHA system is a 157 servo-valve (top of Fig. 1) which controls the fluid dynamics 158 inside the chambers. The spool valve is driven by the electri- 159 cal input current $u$ of a torque motor. The displacement of the 160 spool valve $x_{v}$ together with the load pressure $P_{L}$ controls the 161 fluid dynamic inside two chambers A and B which constitute 162 the hydraulic part of the EHA system. The mechanical part of 163 the EHA system is a cylindrical piston which is modeled as 164 a classical mass-spring system. The position of the cylindrical 165 piston $x_{p}$ obeys to the fundamental principle of dynamics.

\section{A. State-Space Representation of the EHA}

Considering the following states variable: $x=168$ $\left[x_{1}, x_{2}, x_{3}\right]^{T}=\left[x_{p}, \dot{x_{p}}, P_{L}\right]^{T}$, the state-space representa- 169 tion of the EHA system can be written under the following 170 form [26], [29], [31]:

$$
\left\{\begin{array}{l}
\dot{x_{1}}=x_{2} \\
\dot{x_{2}}=-\frac{k}{m} x_{1}-\frac{b}{m} x_{2}+\frac{A_{p}}{m} x_{3} \\
\dot{x_{3}}=-\alpha x_{2}-\beta x_{3}+\gamma \sqrt{P_{s}-\operatorname{sign}(u) x_{3}} u
\end{array}\right.
$$


where $x_{p}$ is the piston position (m). $\dot{x}_{p}(\mathrm{~m} / \mathrm{s})$ is the piston velocity and $P_{L}(\mathrm{~Pa})$ is the pressure load inside the chambers of the hydraulic part. $k$ is the load spring constant $(\mathrm{N} / \mathrm{m}), b$ is the viscous damping coefficient $[\mathrm{N} /(\mathrm{m} / \mathrm{s})]$, and $A_{p}$ is the cylinder bore $\left(\mathrm{m}^{2}\right) . P_{s}$ is the supply pressure (Pa). $\alpha, \beta, \gamma$ are the hydraulic coefficients of the EHA model. These coefficients depend on the flow characteristics of the EHA system. For more details about the expression of the hydraulic coefficients $\alpha, \beta, \gamma$ and the modeling issues of the EHA system, the reader is referred to the work of the authors [26], [29] and their corresponding literature.

\section{B. Modeling Uncertainties and Time-Varying Disturbances Affecting the EHA System}

In [29] and [31], the authors distinguished between two types of disturbances $d_{1}$ and $d_{2}$ which can affect the EHA system. The first one $d_{1}$ is the mechanical disturbance which is the result of lumping together the modeling parametric uncertainties, the load charge $F_{\text {Load }}$, and the friction force $F_{\text {friction }}$ acting on the mechanical part of the EHA system. As reported by the authors in [32], the second term $d_{2}$ does not hold the same significance as $d_{1}$. Indeed, $d_{2}$ represents the parametric deviation over the hydraulic coefficients $\alpha, \beta, \gamma$ and potential leakage affecting the hydraulic device of the EHA system. These parameters are also sensitive to temperature inside the EHA system. Taking into account these issues, the disturbed EHA model can be written as follows [29]:

$$
\left\{\begin{array}{l}
\dot{x_{1}}=x_{2} \\
\dot{x_{2}}=-\frac{k}{m} x_{1}-\frac{b}{m} x_{2}+\frac{A_{p}}{m} x_{3}-\frac{d_{1}}{m} \\
\dot{x_{3}}=-\alpha x_{2}-\beta x_{3}+\gamma \sqrt{P_{s}-\operatorname{sign}(u) x_{3}} u+d_{2}
\end{array}\right.
$$

where $d_{1}(t)$ and $d_{2}(t)$ are expressed as follows [31]:

$$
\begin{aligned}
& d_{1}(t)=-\triangle \frac{k}{m} x_{1}-\triangle \frac{b}{m} x_{2}-\triangle \frac{A_{p}}{m} x_{3}+F_{\text {Load }}+F_{\text {Friction }} \\
& d_{2}(t)=-\triangle \alpha x_{2}-\triangle \beta x_{3}+\triangle \gamma \sqrt{P_{s}-\operatorname{sign}(u) x_{3}} u
\end{aligned}
$$

The $\triangle$ symbolizes the considered parametric uncertainties affecting the mechanical and the hydraulic part of the EHA system. System (2) can be expressed under the following compact form:

$$
\left\{\begin{array}{l}
\dot{x}=A x+\varphi(x, u)+B_{d} d \\
y=C x=x_{1}
\end{array}\right.
$$

where $\boldsymbol{x} \in \mathbf{R}^{3}$ and $y \in \mathbf{R}$ represent, respectively, the state vector and the measured piston position $x_{1}=x_{p}$. The vector $\boldsymbol{u} \in$ $\mathbf{R}$ describes the set of admissible inputs. $d(\boldsymbol{t}) \in \mathbf{R}^{2}$ denotes the vector of the disturbances which affect the EHA. $B_{d}$ with dimensions $3 \times 2$. The matrices $A, B_{d}, C$, and vector $\phi(\boldsymbol{x}, \boldsymbol{u})$ have the following structure:

$$
A=\left(\begin{array}{ccc}
0 & 1 & 0 \\
0 & 0 & \frac{A_{p}}{m} \\
0 & 0 & 0
\end{array}\right)
$$

$$
\begin{aligned}
& B_{d}=\left(\begin{array}{cc}
0 & 0 \\
\frac{-1}{m} & 0 \\
0 & 1
\end{array}\right) \\
& C=\left(\begin{array}{lll}
1 & 0 & 0
\end{array}\right) \\
& \varphi(x, u)=\left(\begin{array}{c}
0 \\
-\frac{k}{m} x_{1}-\frac{b}{m} x_{2} \\
-\alpha x_{2}-\beta x_{3}+\gamma \sqrt{P_{s}-\operatorname{sign}(u) x_{3}} u
\end{array}\right) .
\end{aligned}
$$

\section{Problem Formulation}

For system (4), the piston position is available for measure- 210 ment only at each sampling times $t_{k}$ imposed by the frequency 211 acquisition (the sampling period) of the sensor manufacturer. In 212 this paper, we have to design a robust sampled data observer 213 which simultaneously estimates the unmeasurable states $x_{2}, 214$ $x_{3}$, and the hydraulic disturbance term $d_{2}$ of system (4). The 215 designed observer must deal with the sampling phenomenon of 216 the measured piston position $x_{p}$ and must be robust facing the 217 mechanical disturbance term $d_{1}(t)$. Under these considerations, 218 system (4) is rewritten as follows:

$$
\left\{\begin{array}{l}
\dot{x}=A x+\varphi(x, u)+B_{d} d \\
y\left(t_{k}\right)=C x\left(t_{k}\right)=x_{1}\left(t_{k}\right) .
\end{array}\right.
$$

System (5) combines a continuous dynamic behavior for the 220 states $x_{1}, x_{2}, x_{3}$ between two sampling times $\left[t_{k}, t_{k+1}\right]$ and an 221 updated step for the state $x_{1}$ which occurs at the sampling times 222 $t=t_{k}$.

III. Continuous-Discrete Time-Observer Design 224 FOR THE EHA SYSTEM

In this section, we design a continuous-discrete time 226 observer for the EHA system. Since $d_{2}$ is the main distur- 227 bance term, we use the well-known technique of the augmented 228 state system in order to estimate it. Following this, we add an 229 extended variable $x_{4}=d_{2}$ such as $\dot{x}_{4}=h(t)$ to system (5) so 230 that the augmented state system can be written as follows:

$$
\left\{\begin{array}{l}
\dot{\bar{x}}=\bar{A} \bar{x}+\overline{\varphi(\bar{x}, u)}+\delta(t) \\
y=\bar{C} \bar{x}=x_{1}
\end{array}\right.
$$

where $\bar{x}=\left[x_{1}, x_{2}, x_{3}, x_{4}\right]$ and

$$
\begin{aligned}
\bar{A} & =\left(\begin{array}{cccc}
0 & 1 & 0 & 0 \\
0 & 0 & \frac{A_{p}}{m} & 0 \\
0 & 0 & 0 & 1 \\
0 & 0 & 0 & 0
\end{array}\right) \\
\overline{\varphi(\bar{x}, u)} & =\left(\begin{array}{c}
0 \\
-\alpha x_{2}-\beta x_{3}+\gamma \sqrt{P_{s}-\operatorname{sign}(u) x_{3}} u \\
0
\end{array}\right) \\
\delta(t) & =\left(\begin{array}{c}
0 \\
\frac{-d_{1}}{m} \\
0 \\
h
\end{array}\right) \\
\bar{C} & =\left(\begin{array}{ccc}
1 & 0 & 0
\end{array}\right) .
\end{aligned}
$$




\section{B. Observability Analysis}

From the structure of matrices $\bar{A}, \bar{C}$ in system (6), it can be easily checked that the pair $(\bar{A}, \bar{C})$ is observable. Hence, their exists two matrices $P, Q$ such that the following Lyapunov function is satisfied:

The function $f$ is a saturation function which is introduced to guaranty that the estimated states $\hat{\bar{x}}$ remains inside the compact set so that the Lipschitz constant $\beta_{0}$ always exists. The $\triangle_{\theta}$ is a diagonal matrix $4 \times 4$ defined by

$$
\triangle_{\theta}=\left(\begin{array}{cccc}
1 & 0 & 0 & 0 \\
0 & \frac{1}{\theta} & 0 & 0 \\
0 & 0 & \frac{1}{\theta^{2}} & 0 \\
0 & 0 & 0 & \frac{1}{\theta^{3}}
\end{array}\right)
$$

and the vector gains $K \in \mathbb{R}^{4 \times 1}$ are chosen so that the matrix $(\bar{A}-K \bar{C})$ is Hurwitz. The vector $\hat{\overline{\boldsymbol{x}}}$ is the continuous-time estimate of the system state $\bar{x}$. The vector $\boldsymbol{w}(t)$ represents the prediction of the output between two sampling times. The prediction $\boldsymbol{w}(t)$ is updated (reinitialized) at each sampling instant $t=t_{k}$. where $\mu>0$ is a free-positive constant and $P$ is a symmetric 267 positive definite matrix.

Remark 3: Comparing to the work of the authors in [26], 269 [32], the novelty in the designed observer (8) is the introduc- 270 tion of the intersample output predictor term $w(t)$ [35] in the 271 correction term. The dynamic of this predictor is simply a copy 272 of the dynamics of system states equations. The role of the out- 273 put predictor term is to provide a continuous time prediction of 274 the output measured variable $y(t)$. Indeed, since the measured 275 output variable $y(t)$ is sampled, its values $y\left(t_{k}\right)$ are available 276 for the observer only at sampling times $t=t_{k}$. Comparing to 277 constant-gain zero-order-hold $(\mathrm{ZOH})$ approaches which main- 278 tain $y\left(t_{k}\right)$ constant between the sampling times, the output 279 predictor term $w(t)$ will provide a continuous time estimation 280 of $y(t)$ as it is the case in continuous time-observer design 281 framework.

Now, we are able to state the main results of this paper. 283

Theorem 1: Consider the EHA system (6), and suppose that 284 assumptions (1-2) holds, given a sampling period $T$, choose 285 $\sigma_{0}, \sigma_{1}, \sigma_{2}$ as in (17), define $\sigma_{3}=T e^{\sigma T} \frac{2 \sigma_{1}\left(\theta+\beta_{0}\right)}{\sigma_{0} \sqrt{\lambda_{\min }(P)}}$ then sys- 286 tem (8) is an exponential sampled data observer for system 287 (6) with the following properties: the vector of the observa- 288 tion error $\left\|\bar{e}_{\bar{x}}\right\|$ converges exponentially toward a ball whose 289 radius $R=\frac{2 \sigma_{2}}{\sigma_{0} \sqrt{\lambda_{\min }(P)}\left(1-\sigma_{3}\right)}$. Moreover, there exists a real 290 positive bounded $T_{\max }$ satisfying inequality (34), so that for all 291 $T \in\left(0, T_{\max }\right)$, the radius of the ball can be made as small as 292 desired by choosing large values of $\theta$ and $k_{i=1, \ldots, 4}$. 293

Proof 1: The proof of this theorem 1 is inspired from the 294 work of the authors in [35]. Let us now define the following 295 observer $e_{\bar{x}}$ and the output $e_{w}(t)$ errors as follows:

$$
\left\{\begin{array}{l}
e_{\bar{x}}(t)=\hat{\bar{x}}-\bar{x} \\
e_{w}(t)=w(t)-y(t)=w(t)-\bar{C} \bar{x}
\end{array}\right.
$$

$$
P(\bar{A}-K \bar{C})+(\bar{A}-K \bar{C})^{T} P \leq-\mu \mathbb{I}_{n}
$$

Combining (6) and (8), we can easily check that for the EHA 297 system (6), the following properties are satisfied: $\theta \triangle_{\theta}^{-1} \bar{A} \triangle_{\theta}=298$ $\theta \bar{A}$ and $\triangle_{\theta}^{-1} K \bar{C}=\triangle_{\theta}^{-1} K \bar{C} \triangle_{\theta}$. Introducing the well-known 299 change in coordinate in the high gain literature $\bar{e}_{\bar{x}}=\triangle_{\theta} e_{\bar{x}} \quad 300$ yields the following dynamics of the state and the output errors: 301

$$
\left\{\begin{aligned}
\dot{\bar{e}}_{\bar{x}}= & \theta(\bar{A}-K \bar{C}) \bar{e}_{\bar{x}}+\triangle_{\theta}(\overline{\varphi(f(\hat{\bar{x}}), u)}-\overline{\varphi(\bar{x}, u)}) \\
& +\theta K e_{w}-\triangle_{\theta} \delta(t) \\
\dot{e}_{w}= & \theta \bar{e}_{\bar{x} 2}+\left(\overline{\left.\varphi_{1}(f(\hat{\bar{x}})), u\right)}-\overline{\varphi_{1}(\bar{x}, u)}\right)
\end{aligned}\right.
$$

Let us now consider the following candidate Lyapunov 302 quadratic function $V=\bar{e}_{\bar{x}}^{T} P \bar{e}_{\bar{x}}$ :

$$
\begin{gathered}
\dot{V} \leq-\mu \theta\left\|\bar{e}_{\bar{x}}\right\|^{2}+2 \bar{e}_{\bar{x}}^{T} P \triangle_{\theta}(\overline{\varphi(f(\hat{\bar{x}}), u)}-\overline{\varphi(\bar{x}, u)}) \\
+2 \theta \bar{e}_{\bar{x}}^{T} P K e_{w}(t)-2 \bar{e}_{\bar{x}}^{T} P \triangle_{\theta} \delta
\end{gathered}
$$

Taking into account Assumptions (1-2) we have

$$
\begin{gathered}
\dot{V} \leq-\mu \theta\left\|\bar{e}_{\bar{x}}\right\|^{2}+4 \beta_{0} \lambda_{\max }(P)\left\|\bar{e}_{\bar{x}}\right\|^{2}+2 \theta\|P K\|\left\|\bar{e}_{\bar{x}}\right\|\left|e_{w}(t)\right| \\
+4 \lambda_{\max }(P)\left\|\bar{e}_{\bar{x}}\right\| \xi
\end{gathered}
$$

where $\xi=\sqrt{\mu_{1}^{2}+\mu_{2}^{2}}$. 
306

Using the well-known property

$$
\lambda_{\min }(P)\left\|\bar{e}_{\bar{x}}\right\|^{2} \leq V \leq \lambda_{\max }(P)\left\|\bar{e}_{\bar{x}}\right\|^{2}
$$

307 we derive

$$
\begin{aligned}
& \dot{V} \leq-\mu \theta \frac{V}{\lambda_{\max }(P)}+\frac{4 \beta_{0} \lambda_{\max }(P) V}{\lambda_{\min }(P)} \\
& +2 \theta\|P K\| \sqrt{\frac{V}{\lambda_{\min }(P)}}\left|e_{w}(t)\right|+4 \lambda_{\max }(P) \sqrt{\frac{V}{\lambda_{\min }(P)}} \xi .
\end{aligned}
$$

308 Now choosing the parameter $\theta$ such that $\theta>\theta_{0}$ with $\theta_{0}=$ 309 $\sup \left\{1, \frac{8 \beta_{0} \lambda_{\max }^{2}(P)}{\mu \lambda_{\min }(P)}\right\}$, we have

$$
\begin{gathered}
\dot{V} \leq-\mu \theta \frac{V}{2 \lambda_{\max }(P)}+2 \theta\|P K\| \sqrt{\frac{V}{\lambda_{\min }(P)}}\left|e_{w}(t)\right| \\
+4 \lambda_{\max }(P) \sqrt{\frac{V}{\lambda_{\min }(P)}} \xi .
\end{gathered}
$$

310 Considering now the function $W=\sqrt{V}$, then we obtain

$$
\begin{gathered}
\dot{W} \leq-\mu \theta \frac{W}{4 \lambda_{\max }(P)}+\theta\|P K\| \frac{\left|e_{w}(t)\right|}{\sqrt{\lambda_{\min }(P)}} \\
+2 \frac{\lambda_{\max }(P)}{\sqrt{\lambda_{\min }(P)}} \xi .
\end{gathered}
$$

311 Let us set

$$
\left\{\begin{array}{l}
\sigma_{0}=\frac{\mu \theta}{4 \lambda_{\max }(P)} \\
\sigma_{1}=\frac{\theta\|P K\|}{\sqrt{\lambda_{\min }(P)}} \\
\sigma_{2}=2 \frac{\lambda_{\max }(P)}{\sqrt{\lambda_{\min }(P)}} .
\end{array}\right.
$$

312 Integrating (16), then

$$
\begin{gathered}
W(t) \leq e^{-\sigma_{0}\left(t-t_{0}\right)} W\left(t_{0}\right)+\sigma_{1} e^{-\sigma_{0} t} \int_{t_{0}}^{t} e^{\sigma_{0} s}\left|e_{w}(s)\right| d s \\
+\sigma_{2} e^{-\sigma_{0} t} \int_{t_{0}}^{t} e^{\sigma_{0} s}\|\xi(s)\| d s
\end{gathered}
$$

313 Multiplying both sides of (18) by $e^{\sigma t}$ and using the fact that $314 e^{-\left(\sigma_{0}-\sigma\right) t}<1$ we derive

$$
\begin{aligned}
e^{\sigma t} W(t) & \leq M\left(t_{0}\right)+\sigma_{1} e^{-\left(\sigma_{0}-\sigma\right) t} \int_{t_{0}}^{t} e^{\sigma_{0} s} \mid e_{w}(s \mid d s \\
& +\sigma_{2} e^{-\left(\sigma_{0}-\sigma\right) t} \int_{t_{0}}^{t} e^{\sigma_{0} s}\|\xi(s)\| d s
\end{aligned}
$$

315

where $M\left(t_{0}\right)=e^{\sigma_{0} t_{0}} W\left(t_{0}\right)$.

316

317 or

On the other hand, we have

$e^{\sigma t} W(t) \leq M\left(t_{0}\right)+\sigma_{1} e^{-\left(\sigma_{0}-\sigma\right) t} \int_{t_{0}}^{t} e^{\left(\sigma_{0}-\sigma\right) s} e^{\sigma s} \mid e_{w}(s \mid d s$

$$
+\sigma_{2} e^{-\left(\sigma_{0}-\sigma\right) t} \int_{t_{0}}^{t} e^{\left(\sigma_{0}-\sigma\right) s} e^{\sigma s}\|\xi(s)\| d s
$$

$$
e^{\sigma t} W(t) \leq M\left(t_{0}\right)
$$

$$
\begin{aligned}
& +\sigma_{1} e^{-\left(\sigma_{0}-\sigma\right) t}\left(\int_{t_{0}}^{t} e^{\left(\sigma_{0}-\sigma\right) s} d s\right) \sup _{t_{0} \leq s \leq t}\left(e^{\sigma s}\left\|e_{w}(s)\right\|\right) \\
& +\sigma_{2} e^{-\left(\sigma_{0}-\sigma\right) t}\left(\int_{t_{0}}^{t} e^{\left(\sigma_{0}-\sigma\right) s} d s\right) \sup _{t_{0} \leq s \leq t}\left(e^{\sigma s}\|\xi(s)\|\right)
\end{aligned}
$$

which leads to

$$
\begin{aligned}
e^{\sigma t} W(t) \leq & M\left(t_{0}\right) \\
& +\frac{\sigma_{1}}{\sigma_{0}-\sigma} \sup _{t_{0} \leq s \leq t}\left(e^{\sigma s}\left|e_{w}(s)\right|\right) \\
& +\frac{\sigma_{2}}{\sigma_{0}-\sigma} \sup _{t_{0} \leq s \leq t}\left(e^{\sigma s}|| \xi(s) \|\right) .
\end{aligned}
$$

Now taking $0<\sigma<\sigma_{0} / 2$, we derive

$$
\begin{aligned}
\sup _{t_{0} \leq s \leq t}\left(e^{\sigma s} W(s)\right) & \leq M\left(t_{0}\right) \\
& +2 \frac{\sigma_{1}}{\sigma_{0}} \sup _{t_{0} \leq s \leq t}\left(e^{\sigma s}\left|e_{w}(s)\right|\right) \\
& +2 \frac{\sigma_{2}}{\sigma_{0}} \sup _{t_{0} \leq s \leq t}\left(e^{\sigma s}|| \xi(s) \|\right)
\end{aligned}
$$

and

$$
\begin{gathered}
W(t) \leq e^{-\sigma t} M\left(t_{0}\right)+2 \frac{\sigma_{1}}{\sigma_{0}} \sup _{t_{0} \leq s \leq t}\left(e^{-\sigma(t-s)}\left|e_{w}(s)\right|\right) \\
+2 \frac{\sigma_{2}}{\sigma_{0}} \sup _{t_{0} \leq s \leq t}\left(e^{-\sigma(t-s)}\|\xi(s)\|\right)
\end{gathered}
$$

which leads to

$$
\begin{aligned}
\left\|\bar{e}_{\bar{x}}\right\| & \leq e^{-\sigma t} \frac{M\left(t_{0}\right)}{\sqrt{\lambda_{\min }(P)}} \\
& +\frac{2 \sigma_{1}}{\sigma_{0} \sqrt{\lambda_{\min }(P)}} \sup _{t_{0} \leq s \leq t}\left(e^{-\sigma(t-s)} \mid e_{w}(s \mid)\right. \\
& +\frac{2 \sigma_{2}}{\sigma_{0} \sqrt{\lambda_{\min }(P)}} \sup _{t_{0} \leq s \leq t}\left(e^{-\sigma(t-s)}\|\xi(s)\|\right)
\end{aligned}
$$

and

$$
\begin{aligned}
\sup _{t_{0} \leq s \leq t}\left(e^{\sigma s}\left\|\bar{e}_{\bar{x}}\right\|\right) & \leq \frac{M\left(t_{0}\right)}{\sqrt{\lambda_{\min }(P)}} \\
& +\frac{2 \sigma_{1}}{\sigma_{0} \sqrt{\lambda_{\min }(P)}} \sup _{t_{0} \leq s \leq t}\left(e^{\sigma s}\left|e_{w}(s)\right|\right) \\
& +\frac{2 \sigma_{2}}{\sigma_{0} \sqrt{\lambda_{\min }(P)}} \sup _{t_{0} \leq s \leq t}\left(e^{\sigma(s)}|| \xi(s) \|\right) .
\end{aligned}
$$

On the other hand, we have from (10) the following expression 323 of $\left|e_{w}(t)\right|$ :

$$
\left|e_{w}(t)\right|=\int_{t_{k}}^{t}\left|\theta \bar{e}_{\bar{x} 2}+\left(\overline{\varphi_{1}(f(\hat{\bar{x}}), u)}-\overline{\varphi_{1}(\bar{x}, u)}\right)\right| d s .
$$

Multiplying again both sides of (28) by $e^{\sigma t}$ and taking into 325 account assumptions $1-2$, we have

$$
e^{\sigma t}\left|e_{w}(t)\right| \leq e^{\sigma t}\left(\theta+\beta_{0}\right) \int_{t_{k}}^{t} e^{-\sigma s} e^{\sigma s}\left\|\bar{e}_{\bar{x}}(s)\right\| d s
$$

which leads to

$$
\begin{array}{r}
e^{\sigma t}\left|e_{w}(t)\right| \leq e^{\sigma t}\left(\theta+\beta_{0}\right)\left(\int_{t_{k}}^{t} e^{-\sigma s} d s\right) \\
\sup _{t_{k} \leq s \leq t}\left(e^{\sigma s}\left\|\bar{e}_{\bar{x}}(s)\right\|\right) d s
\end{array}
$$




$$
\begin{aligned}
& \sup _{t_{k} \leq s \leq t} e^{\sigma s}\left|e_{w}(s)\right| \leq T e^{\sigma T}\left(\theta+\beta_{0}\right) \\
& \sup _{t_{k} \leq s \leq t}\left(e^{\sigma s}\left\|\bar{e}_{\bar{x}}(s)\right\|\right) d s
\end{aligned}
$$

329 since $\sup _{t_{k} \leq s \leq t}\left(e^{\sigma s} \| \bar{e}_{\bar{x}}(s)\right) \leq \sup _{t_{0} \leq s \leq t}\left(e^{\sigma s} \| \bar{e}_{\bar{x}}(s)\right) \quad$ and taking into account that $t>t_{0}, t_{1}, \ldots, t_{k}$ we derive that

$$
\begin{aligned}
& \sup _{t_{0} \leq s \leq t} e^{\sigma s}\left|e_{w}(s)\right| \leq T e^{\sigma T}\left(\theta+\beta_{0}\right) \\
& \sup _{t_{0} \leq s \leq t}\left(e^{\sigma s}\left\|\bar{e}_{\bar{x}}(s)\right\|\right) d s
\end{aligned}
$$

Combining (32) with (27) we have

$$
\begin{aligned}
& \sup _{t_{0} \leq s \leq t}\left(e^{\sigma s}\left\|\bar{e}_{\bar{x}}\right\|\right) \leq \frac{M\left(t_{0}\right)}{\sqrt{\lambda_{\min }(P)}} \\
& \left.+T e^{\sigma T} \frac{2 \sigma_{1}}{\sigma_{0} \sqrt{\lambda_{\min }(P)}}\left(\theta+\beta_{0}\right) \sup _{t_{0} \leq s \leq t}\left(e^{\sigma s}\left\|\bar{e}_{\bar{x}}(s)\right\|\right) d s\right) \\
& +\frac{2 \sigma_{2}}{\sigma_{0} \sqrt{\lambda_{\min }(P)}} \sup _{t_{0} \leq s \leq t}\left(e^{\sigma(s)}\|\xi(s)\|\right)
\end{aligned}
$$

setting $\sigma_{3}=T e^{\sigma T} \frac{2 \sigma_{1}\left(\theta+\beta_{0}\right)}{\sigma_{0} \sqrt{\lambda_{\min }(P)}}$ then selecting $T_{\max }$ satisfying the following the small gain condition:

$$
T_{\max } e^{\sigma T_{\max }} \frac{2 \sigma_{1}\left(\theta+\beta_{0}\right)}{\sigma_{0} \sqrt{\lambda_{\min }(P)}}<1
$$

we have

$$
\begin{gathered}
\left\|\bar{e}_{\bar{x}}\right\| \leq e^{-\sigma t} \frac{M\left(t_{0}\right)}{\sqrt{\lambda_{\min }(P)}\left(1-\sigma_{3}\right)} \\
\left.+\frac{2 \sigma_{2}}{\sigma_{0} \sqrt{\lambda_{\min }(P)}\left(1-\sigma_{3}\right)} \sup _{t_{0} \leq s \leq t}\|\xi(s)\|\right) .
\end{gathered}
$$

This complete the proof of Theorem 1.

Remark 4: Contrary to ([34], (35) demonstrates the global exponential convergence of the vector of the observation error $\left\|\bar{e}_{\bar{x}}\right\|$ toward a ball whose radius depends on the magnitude of the disturbance vector $\boldsymbol{\xi}$. In addition, the maximum sampling period $T_{\max }$ derived in (34) is less restrictive comparing to the one derived in [34] which depends on the computation of a bounded positive function $\psi(t)$ (see (13) in [34]).

Remark 5: The radius of the ball $R$ is defined such that $R=$ $\frac{2 \sigma_{2}}{\sigma_{0} \sqrt{\lambda_{\min }(P)}\left(1-\sigma_{3}\right)}$. We also notice that in the case where there is no mechanical disturbances (i.e., $d_{1}=0$ ) and the hydraulic disturbances are constant or equal to 0 , we have an exponential convergence of the observation error $\left\|\bar{e}_{\bar{x}}\right\|$ toward 0 . Looking at the expression of the maximum sampling period $T_{\max }$ in (34), we can easily see that when $\sigma$ tends to zero, $T_{\max } \simeq \frac{1}{\theta}$. Hence, augmenting $\theta$ will diminish the value of $T_{\max }$. On the other hand, large values of parameter $\theta$ will contribute to reduce the radius $R$ and hence to improve the performance of our observer. However, it is well known that the high gain observers literature, augmenting the values of $\theta$ will lead to the undesirable peaking phenomenon which consists in an impulsive behavior of the states estimation trajectory around initial conditions.
TABLE I

Numerical Parameter Values for the Eha System

\begin{tabular}{|c|c|}
\hline Parameters & Value \\
\hline$m$ & 0.5 \\
\hline$b$ & 0 \\
\hline$k$ & $5.651110 \times 10^{5}$ \\
\hline$A_{p}$ & $5.058 \times 10^{-4}$ \\
\hline$k_{v}$ & $1.333 \times 10^{-5}$ \\
\hline$\alpha$ & $3.257 \times 10^{10}$ \\
\hline$\beta$ & 2.146 \\
\hline$\gamma$ & $7.169 \times 10^{9}$ \\
\hline$P_{s}$ & $2.1 \times 10^{7}$ \\
\hline
\end{tabular}

TABLE II

$\mathrm{T} 2: 1$

Parameters of the Hybrid OBServer

\begin{tabular}{|c||c|c|c|}
\hline Parameter & $\theta$ & $K=\left(\begin{array}{c}K_{1} \\
K_{2} \\
K_{3} \\
K_{4}\end{array}\right)$ & $T_{s}$ \\
\hline
\end{tabular}

IV. Simulations and Experimental Results

A. Numerical Simulation of the Hybrid Observer Coupled 358 With PI Controller for the EHA System Subject to 359 Mechanical and Hydraulic Disturbances

The performance of the proposed observer will be evaluated 361 first under MATLAB/Simulink Software. For the purpose of 362 comparison, the numerical simulations were performed on the 363 EHA system validated experimentally by the authors in [26] 364 and [29]. The model parameters' values are shown in Table I. 365

In this numerical simulations, we will demonstrate the 366 effectiveness of our proposed observer in terms of states/ 367 disturbances estimation and positioning control. In [29], the 368 authors considered a sinusoidal reference position signal $x_{1 d}=369$ $0.008 \sin (2 \pi t)$. For the purpose of tracking $x_{1 d}$, a PI controller 370 was employed and combined with the proposed observer (8) so 371 that the novel PI control law $u$ is expressed as follows:

$$
u=K_{p}\left(w(t)-x_{1 d}\right)+K_{i} \int\left(w(t)-x_{1 d}\right)
$$

where $x_{1}=x_{p}$ is the piston position and $K_{p}=3.18 \times 373$ $10^{-2}, K_{i}=100$ are the PI gains. The PI controller gains were 374 tuned in order to track. The numerical simulations were per- 375 formed using the Runge-Kutta solver with a fixed step size 376 $T_{\text {sim }}=10^{-4}$ s. The parameters of the hybrid observer are sum- 377 marized in Table II where $T_{s}$ is the sampling period of our 378 proposed hybrid (continuous-discrete time) observer.

The values of the observer parameters used in this simulation 380 are $\theta=1000, K=(10,35,49426,23724)$ and $T_{s}=1 \mathrm{~ms} . \quad 381$

The evaluation of our observer is performed under the con- 382 sideration that both mechanical and hydraulic disturbances 383 affect the considered EHA system in this paper. For the 384 mechanical disturbance term $d_{1}$, we have taken the same one 385 considered by the authors [29]. To show the robustness of our 386 observer facing the mechanical disturbances, we considered 387 it in the simulation not from the beginning but at $t=10 \mathrm{~s} .388$ Hence, the term $d_{1}$ in the disturbed model of the EHA in (2) 389 

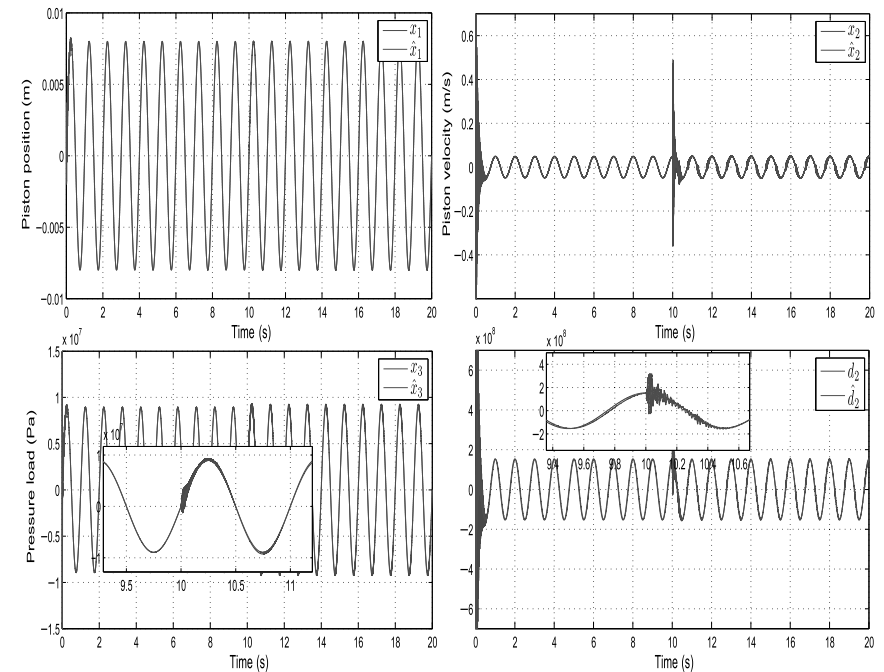

F2:1 Fig. 2. Estimation of $x_{1}, x_{2}, x_{3}, d_{2}$ for $\theta=1000$ and $T_{s}=1 \mathrm{~ms}$ with F2:2 mechanical and hydraulic disturbances. is expressed as follows:

$$
d_{1}(t)= \begin{cases}0, & \text { if } t<10 \mathrm{~s} \\ 294 \sin \left(62.83 x_{1}\right)+20 \operatorname{sign}\left(x_{2}\right), & \text { if } t \geq 10 \mathrm{~s}\end{cases}
$$

We also assume in this simulation that $10 \%$ additive parametric variation affects the hydraulic coefficients $\gamma$; hence (see Section II), the hydraulic disturbance term $d_{2}$ takes the following form:

$$
d_{2}(t)=10 \% \sqrt{P_{s}-\operatorname{sign}(u) x_{3}} u .
$$

From Fig. 2, we can see that the tracking performance of the reference $x_{1 d}$ even in the presence of the mechanical disturbance at $t=10 \mathrm{~s}$ is achieved correctly by the PI controller (36). The robustness of the PI controller facing the mechanical disturbance can be also seen in Fig. 2 where we can see that this disturbance has no effect on the tracking performance of the motion reference trajectory $x_{1 d}$. For the estimation of the piston velocity $x_{2}$, the pressure load $x_{3}$, and the hydraulic disturbance term $d_{2}$, we can see the effect of the mechanical disturbance (see Fig. 2 top right, bottom left, and right) which consists in a deviation of the states estimation trajectory occurring at $t=10 \mathrm{~s}$. Meanwhile, this deviation is quickly rejected by the observer, thanks to the large value of parameter $\theta$ taken in this simulation. As mentioned in Remark 5, large values of parameter $\theta$ will lead to a better rejection of the mechanical and the hydraulic disturbance term, however, this will amplify the peaking phenomenon which consists in an impulsive behavior of the trajectory of the states estimation at the beginning of the simulation (see Fig. 2).

\section{B. Performance Comparison With the Observer Designed in [26] and [32]}

To show the performance of our proposed observer, we have performed a comparison with the observers designed in [26] and [32]. Indeed, the observers [26], [32] have the same high gain like observer structure as the one considered in the design
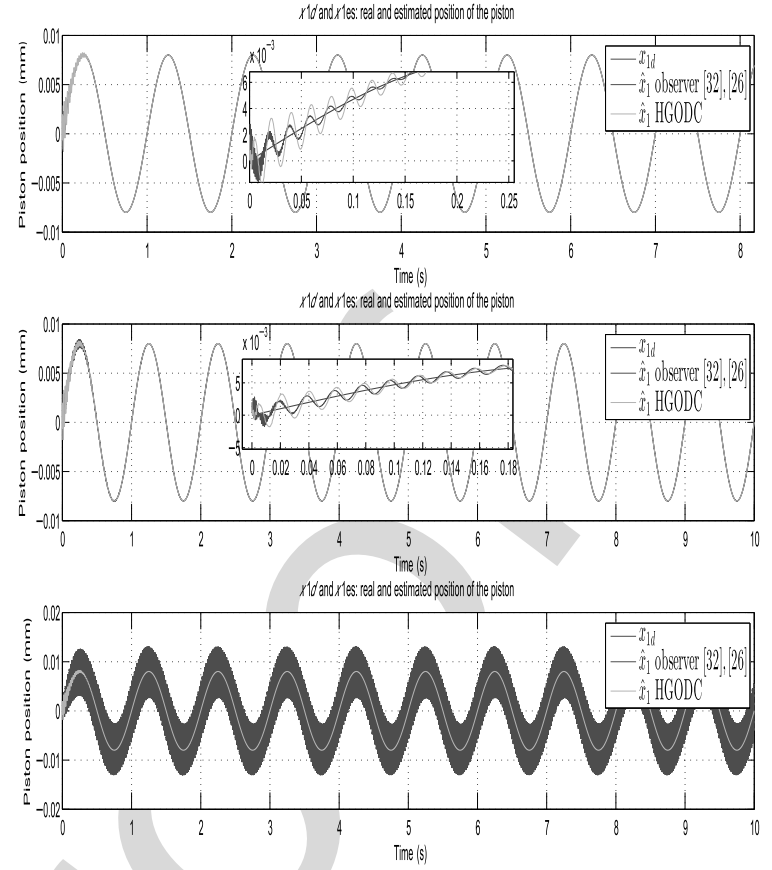

Fig. 3. Comparison of position tracking performance between our F3:1 observer [high gain observer discrete-continuous (HGODC)] $\left(T_{s}=\mathrm{F} 3: 2\right.$ $1 \mathrm{~ms}$ ) and observers [26], [32] (top: $T_{s}=0.1 \mathrm{~ms}$; middle: $T_{s}=0.5 \mathrm{~ms} ; \mathrm{F} 3: 3$ bottom: $T_{s}=1 \mathrm{~ms}$ ).

$\mathrm{F} 3: 4$

of our observer. By taking into account the sampling effect in 420 the structure of these two observers, a continuous-discrete time 421 version of the observers designed in [26] and [32] can be written 422 as follows:

423

$$
\dot{\bar{x}}=\bar{A} \hat{\bar{x}}+\overline{\varphi(f(\hat{\bar{x}}), u)}-H\left(\bar{C} \hat{\bar{x}}(t)-y\left(t_{k}\right)\right) .
$$

We notice that in the case of our observer $H=\theta \triangle_{\theta}^{-1} K$. The 424 structure of (37) uses the sampled data $y\left(t_{k}\right)$ in the correction 425 term since that continuous measured variable $y(t)$ is available 426 only at sampled instants $t=t_{k}$. The simulations presented in 427 Fig. 3 show the performance of observer (8) and observer (37) 428 in terms of position tracking performances. For our proposed 429 observer (named HGODC), we have fixed the value of $T_{s}$ to 430 $1 \mathrm{~ms}$. For observer (37), three values were taken $\left(T_{s}=0.1,431\right.$ 0.5 , and $1 \mathrm{~ms}$ ). Looking at Fig. 3 (top), we can see that even 432 if observer (37) performs better in the transitory regime, our 433 observer has quite the same performance. Recalling that in this 434 case, $T_{s}=0.1 \mathrm{~ms}$ for observer (37) which is the same sampling 435 period as the one of the solver, we can say that our observer 436 recovers the performances of continuous time observers. When 437 augmenting the sampling period of observer (37) to $0.5 \mathrm{~ms}, 438$ we can see that for observer (37), the performance degrades. 439 Finally, when the two observers have the same sampling peri- 440 ods ( $\left.T_{s}=1 \mathrm{~ms}\right)$, observer (37) diverges and the PID controller, 441 which is based on the estimation provided by observer (37), 442 fails to track the desired trajectory $x_{1 d}$.

\section{Experimental Validation}

To illustrate the performance of our proposed observer, an 445 experimental test rig platform has been set up and photographed 446 


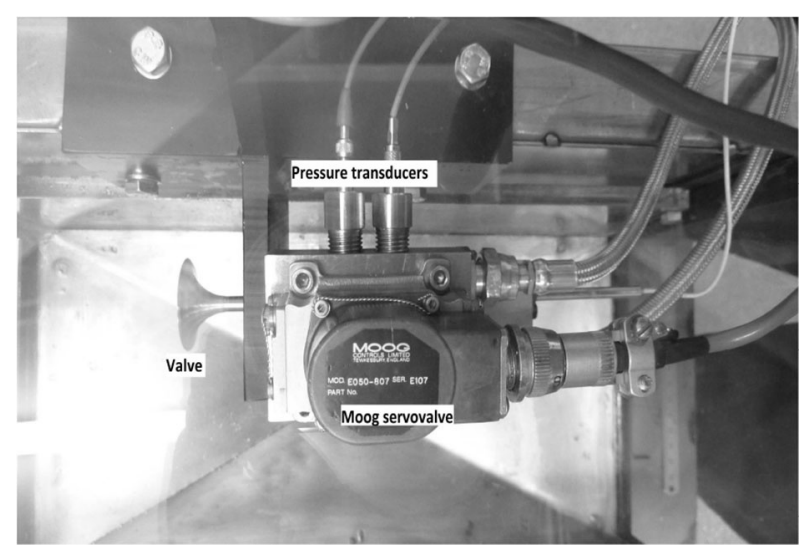

F4:1 Fig. 4. Moog servo-valve and the EHA actuator assembly.

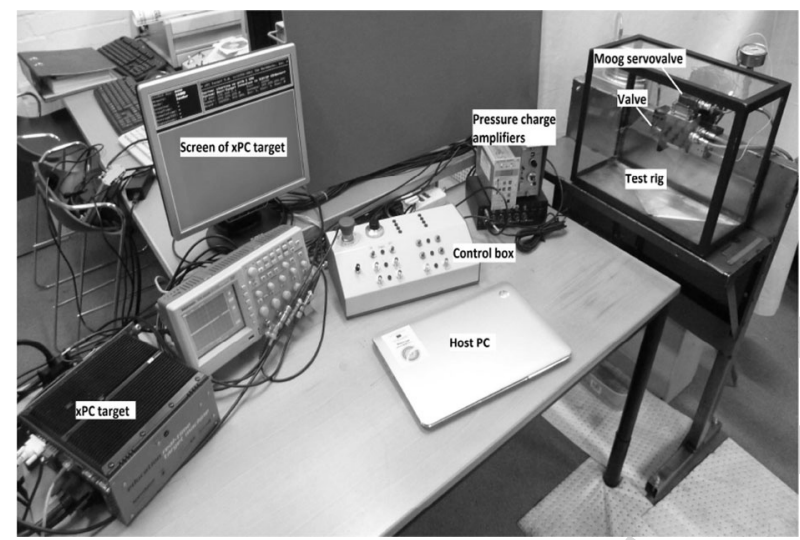

F5:1 Fig. 5. Control system of the experimental test rig of the EHA system.

in Figs. 4 and 5. The test rig was constructed in the Brighton University to investigate the performance of the EHA assembly and the control parameters influencing the motion of the poppet valve. The test rig comprised of three main subsystems: a hydraulic oil pressure supply; a hydraulic valve actuation assembly; and the servo-valve control signal and valve position interface.

Hydraulic oil from a large tank was supplied to a smaller reservoir coupled to a high-pressure pump and accumulator. An electromagnetic pressure-limit switch was used to regulate the supply of high-pressure oil to the hydraulic valve actuation assembly via an oil filter. The supply pressure was regulated to 70 bar \pm 2 bar by a pressure-limit switch.

The actuator body housed a double-acting hydraulic piston, oil-sealing end plates, and the high-pressure oil supply and return feed lines. A continuous-proportional (four-way) directional servo-valve (Moog series 31) was used to control the flow rate of hydraulic oil to the hydraulic piston by means of a proportional electromagnetic servo control signal. The interchangeable poppet valve head was attached to one end of the hydraulic piston and a linear variable differential transducer (LVDT) was mounted to the opposite end to record the change in valve position. The calibration factor for the amplified output of the LVDT sensor (Lord MicroStrain) was $2.97 \mathrm{~mm} / \mathrm{V} \pm 0.005 \mathrm{~mm} / \mathrm{V}$. Two piezoelectric gauge pressure
TABLE III

EHA Parameter Values for the Experimental Test Rig

\begin{tabular}{|c|c|}
\hline Parameters & Value \\
\hline$m$ & 0.05 \\
\hline$k$ & 2000 \\
\hline$b$ & 0.1398 \\
\hline$A_{p}$ & 0.0614 \\
\hline$k_{v}$ & 0.02 \\
\hline$\alpha$ & 28.2226 \\
\hline$\beta$ & 0.0063 \\
\hline$\gamma$ & 0.0029 \\
\hline$P_{s}$ & $7 \times 10^{6}$ \\
\hline
\end{tabular}

transducers (Kistler type 6125 transducer and type 5011 ampli- 472 fier) were used to measure the instantaneous and difference in 473 oil pressures in the supply and return chambers either sides of 474 the hydraulic piston. The pressure transducer was calibrated to 475 $20 \mathrm{bar} / \mathrm{V}$. The full-scale error in the transducer was \pm 3 bar. The 476 value of the oil pressure at the instant of initial piston motion 477 was used as the gauge reference pressure.

478

The control system for the electro-hydraulic valve system 479 was based on a real-time simulation and testing platform 480 (hardware in the loop, HIL); MathWorks MATLAB Simulink 481 and XPC Target application and a real-time target machine 482 (Speedgoat $\mathrm{GmbH}$ ). Positional feedback of the valve was deter- 483 mined from the LVDT sensor output. The actuation of the 484 directional servo-valve was achieved using a current driver sig- 485 nal rated to $\pm 50 \mathrm{~mA}$. The displacement of the poppet valve is 486 comprised between [20-32] mm. Based on the physical param- 487 eters of the experimental test rig [36], the nominal values of the 488 EHA model parameters were identified and listed in Table III. 489

In the following experiments, the parameters' values of 490 the hybrid observer for this experiment are $\theta=500, K=491$ $(2.8,2.87,1.0423,0.1710)$, and $T_{s}=1 \mathrm{~ms}$.

\section{PID Control Design for the Experimental Test Rig}

In order to track the motion reference $x_{1 d}$, the following 494 PID control law $u$ with a velocity feedforward action was 495 implemented

$$
\begin{gathered}
u=K_{p}\left(x_{1 d}-w(t)\right)+K_{i} \int\left(x_{1 d}-w(t)\right) \\
+K_{d} \frac{d}{d t}\left(x_{1 d}-w(t)\right)+K_{f} \dot{x}_{1 d}
\end{gathered}
$$

where $K_{p}=0.54, K_{i}=1.93, K_{d}=0.04, K_{f}=1$. As it was 497 the case in the simulation section, the implemented control law 498 $u$ contains the output prediction term $w(t)$. We mention that for 499 this experimental validation, we used the same Runge-Kutta 500 solver with the same fixed step size $T_{\text {sim }}=10^{-4}$ as in the 501 numerical simulations section. The experimental validation was 502 conducted with a sampling period $T_{s}=1 \mathrm{~ms}$ which is 10 times 503 bigger than the fixed step size of the solver.

\section{E. Experimental Performances of the Hybrid Observer 505 Without Disturbance

In this section, we investigate the performance of the hybrid 507 observer for state estimation and piston position tracking 508 

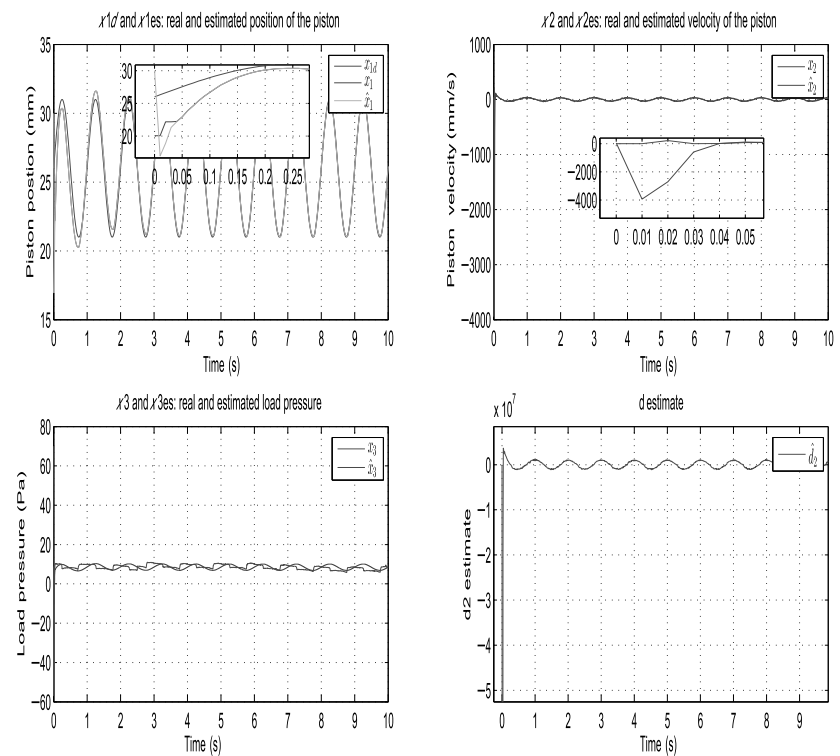

F6:1 Fig. 6. Estimation and tracking performance of $x_{1}, x_{2}, x_{3}, d_{2}$ for F6:2 $\theta=500, T_{s}=1 \mathrm{~ms}$ for EHA system without disturbances.

motion trajectory $x_{1 d}=26+5 \sin (2 \pi t)$. Since the considered EHA system does not drive any mechanical load, we have theoretically $d_{1} \simeq=0$. We also mention that we have used the same nominal values of the EHA system when implementing the hybrid observer.

In Fig. 6 (top left), we show the performance of the hybrid observer in terms of tracking performances and state estimation of the piston position $x_{1}$. We can see in Fig. 6 (top left) that both the tracking performance and the state estimation are achieved correctly by the hybrid observer. For the state estimation of the piston position $x_{1}$, the convergence of the hybrid observer is achieved with small convergence rate [less than $0.05 \mathrm{~s}$ when looking to the zoom of Fig. 6 (top left)]. We can see also that the tracking performance of the motion reference $x_{1 d}$ by the PI controller, which uses the output predictor $w(t)$, is also achieved correctly.

Fig. 6 (top right) shows the state estimation of the piston velocity $x_{2}$. We can see in Fig. 6 (top right) that our hybrid observer provides a very good estimation of the real piston velocity $x_{2}$. A quick look to Fig. 6 (top right) shows that the effect noise, which comes from the numerical differentiation used to obtain the real piston, has been attenuated by our hybrid observer.

In Fig. 6 (bottom left), we present the estimation results of the hydraulic pressure state $x_{3}$ by our proposed observer. First, we can observe from Fig. 6 (bottom left) that our observer provides a good estimation of the hydraulic pressure state $x_{3}$ despite the variations in the hydraulic parameters and the hydraulic disturbance which affects the functioning of the EHA system. The effects of these disturbances can be viewed. In Fig. 6 (bottom right) where we can see that even if there is no mechanical load driven by the EHA system, the estimated disturbance term $\hat{d}_{2}$ is not equal to 0 . Indeed, the difficulty of capturing the hydraulic parameters $(\alpha, \beta, \gamma)$ and the internal leakage occurring on the EHA system generates automatically the disturbance term $d_{2}$. For the reader, we mention that it was
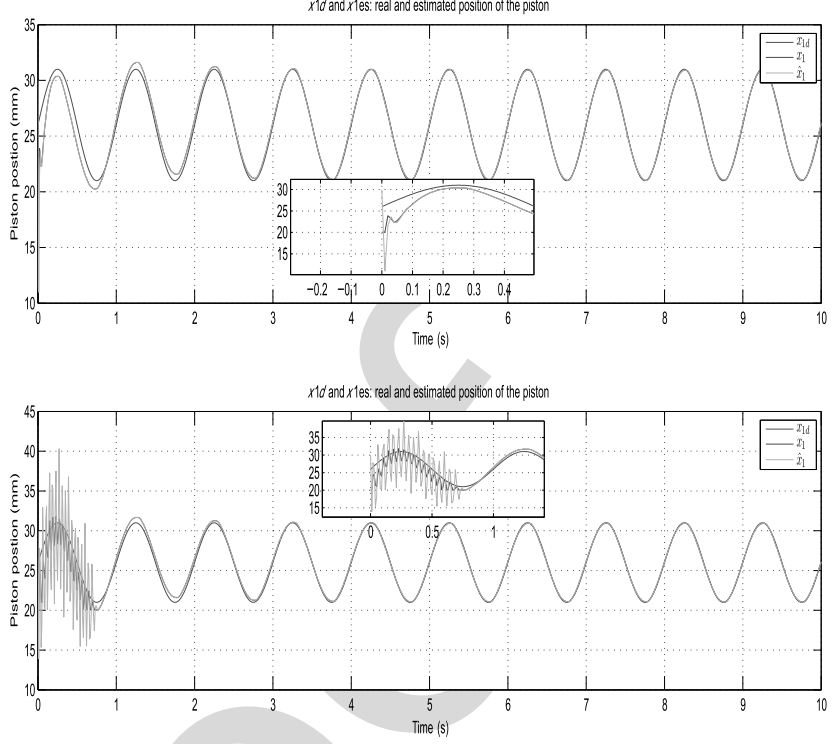

Fig. 7. Estimation and tracking performance of $x_{1}$ for $\theta=500$. (Top) F7:1 $T_{s}=2 \mathrm{~ms}$. (Bottom) $T_{s}=3 \mathrm{~ms}$.

very difficult for us to plot in Fig. 6 (bottom right) the real 545 hydraulic disturbance term $d_{2}$ for the reasons explained above. 546 Finally, we can observe in Fig. 6 (bottom left) that there is 547 small phase lag between the real and the estimated hydraulic 548 pressure $x_{3}$. This observation is quite interesting because of the 549 discrepancies between the numerical simulations and the exper- 550 imental validation of our observer. This discrepancies come 551 from the difficulty of capturing exactly the hydraulic parame- 552 ters of the EHA system and the fact that the dynamic of the 553 electrical part of the EHA system has been neglected in the 554 EHA model. In addition, it appears that the PID control is not 555 able to compensate it. Taking into account that the kistler pres- 556 sure transducers give a relative and not an absolute pressures 557 values in each chamber of the hydraulic actuator, we can say 558 that the estimated hydraulic pressures provided by our observer 559 are good.

\section{F. Effect of the Sampling Period on the Performance of 561 the Hybrid Observer

To compute the maximum allowable sampling period $T_{\max } 563$ of the hybrid observer, we can proceed following two pos- 564 sible manners. The first one is to compute $T_{\max }$ analytically 565 using the expression in (34); however, this will necessitate to 566 know the constant $\beta_{0}$ which is practically very difficult to deter- 567 mine. The second one is to start with a sampling period $T_{s}$ and 568 increasing it until the observer diverges. We proceed follow- 569 ing the second manner. In Fig. 7, we present the experimental 570 results of the estimated piston position $x_{1}$ and the tracking per- 571 formance of the piston position reference $x_{1 d}$. We mention that 572 we did not report the experimental results concerning the esti- 573 mations of the piston velocity $x_{2}$, the hydraulic pressure $x_{3}, 574$ and the hydraulic disturbances $d_{2}$. The reason is that they are 575 characterized by the same dynamic behavior as the results pre- 576 sented in Fig. 7. When increasing $T_{s}$ to $2 \mathrm{~ms}$, we can observe 577 from the top of Fig. 7 that the estimated piston position and 578 

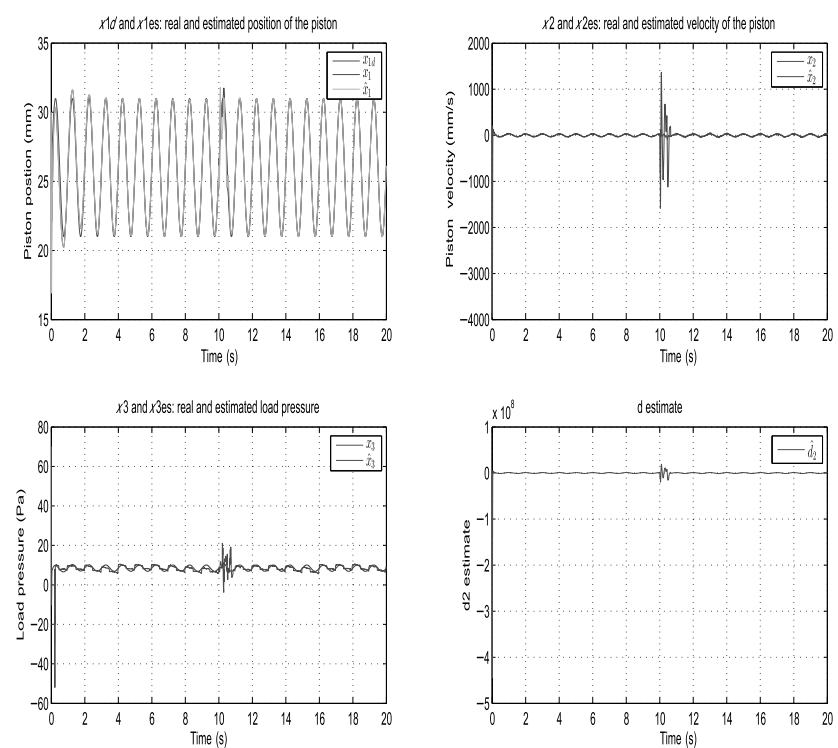

F8:1 Fig. 8. Estimation and tracking performance of $x_{1}, x_{2}, x_{3}, d_{2}$ for F8:2 $\theta=500, T_{s}=1 \mathrm{~ms}$ for EHA system with disturbances.

the tracking performance are quite the same as it is the case of $T_{s}=1 \mathrm{~ms}$. The difference concerns the convergence speed which is slower in the case of $T_{s}=1 \mathrm{~ms}$. When increasing $T_{s}$ to $3 \mathrm{~ms}$, we can observe that the performances of the hybrid observer are affected only in the transitory regime (see bottom of Fig. 7). Indeed, the oscillations observed in the bottom of Fig. 7 are due to the increase in the sampling period $T_{\max }$ to $3 \mathrm{~ms}$ which clearly affects the transitory regime for our hybrid observer. In the permanent regime, the hybrid observer which provides the output predictor term $w(t)$ for the PID controller performs well in the case of estimation and the tracking performance. From this, we can deduce that in the case of this experimental results, $T_{\max } \simeq 2 \mathrm{~ms}$.

\section{G. Experimental Performances of the Hybrid Observer With Disturbance}

To investigate the performance of our observer in the presence of disturbance, an additional disturbance term $d_{3}=2 x_{1 d}$ is inserted in the control input at $t=10 \mathrm{~s}$; meanwhile, the new control input sent to the control board is $u 1=u+2 x_{1 d}$, where $u$ is the previous control calculated by the PID controller. According to the structure of the model of the EHA system, this disturbance will be added to the previously hydraulic disturbance term $d_{2}$ and will change the dynamic of the states $\left(x_{1}, x_{2}, x_{3}, x_{4}\right)$ of the EHA system. We can see from Fig. 8 that both tracking performances and states estimation are achieved correctly by our observer. At $t=10 \mathrm{~s}$, we can see the influence of the disturbances on the performances of our observer. Despite its occurrence, we can clearly say that: first, the PID controller is robust facing this disturbance; since that the PID control law $u$ uses the predictor term $w(t)$ provided by our observer, this will demonstrate the easiness of the incorporation of our observer in a control scheme; second, our observer succeeds to estimate the states and the disturbances affecting the EHA system after $(t=10 \mathrm{~s})$.

\section{CONCLUSION AND FutuRE WORK}

In this paper, a continuous-discrete time observer is designed 614 for the EHAs system subject to discrete time measurement and 615 mechanical and hydraulic disturbances. The exponential con- 616 vergence of the proposed observer is proven using a classical 617 quadratic Lyapunov function based on small gain arguments. 618 The proposed observer is combined with PID controller for the 619 purpose of tracking motion reference trajectory of the piston 620 position for the EHA system. The simulation results and the 621 experimental validation of our proposed observer demonstrate 622 its efficiency in terms of tracking performance and distur- 623 bance estimation. In our future works, we plan to synthesize an 624 output feedback controllers based on the designed continuous- 625 discrete time observer in this paper. The resulting controllers 626 will improve the positioning control for the EHAs system.

\section{REFERENCES}

[1] J. Yao, Z. Jiao, and S. Han, "Friction compensation for low velocity 629 control of hydraulic flight motion simulator: A simple adaptive robust 630 approach," Chin. J. Aeronaut., vol. 26, no. 3, pp. 841-822, Jun. 2013.631

[2] J. Yao, Z. Jiao, B. Yao, Y. Shang, and W. Dong, "Nonlinear adaptive 632 robust control of electrohydraulic load simulator," Chin. J. Aeronaut., 633 vol. 25, no. 5, pp. 766-775, Oct. 2012.

[3] W. Sun, H. Gao, and O. Kaynak, "Adaptive backstepping control for 635 active suspension systems with hard constraints," IEEE/ASME Trans. 636 Mechatronics, vol. 18, no. 3, pp. 1072-1079, Jun. 2013.

[4] Y. Pi and X. Wang, "Observer-based cascade control of a 6-DOF parallel 638 hydraulic manipulator in joint space coordinate," Mechatronics, vol. 20, 639 no. 6, pp. 648-655, Sep. 2010.

[5] W. Sun, Y. Zhao, J. Li, L. Zhang, and H. Gao, "Active suspension control 641 with frequency band constraints and actuator input delay," IEEE Trans. 642 Ind. Electron., vol. 59, no. 1, pp. 530-537, Jan. 2012.

[6] J. Yao, Z. Jiao, D. Ma, and L. Yan, "High-accur of hydraulic rotary actuators with modeling uncertainties," IEEE/ASME 645 Trans. Mechatronics, vol. 19, no. 2, pp. 633-641, Apr. 2014.

[7] H. A. Mintsa, R. Venugopal, J.-P. Kenne, and C. Belleau, "Feedback 647 linearization-based position control of an electrohydraulic servo system 648 with supply pressure uncertainty," IEEE Trans. Control Syst. Technol., 649 vol. 20, no. 4, pp. 1092-1099, Jul. 2012.

[8] H. E. Merritt, Hydraulic Control Systems. Hoboken, NJ, USA: Wiley, 651 1967.

[9] J. Yao, G. Yang, and D. Ma, "Internal leakage fault detection and tolerant control of single-rod hydraulic actuators," Math. Prob. Eng., vol. 2014.

[10] J.-H. Kwon, T.-H. Kim, J.-S. Jang, and I.-S. Lee, "Feedback linearization control of a hydraulic servo system," in Proc. SICE-ICASE Int. Joint Conf., 2006, pp. 455-460.

[11] H.-M. Chen, J.-C. Renn, and J.-P. Su, "Sliding mode control with varying 658 boundary layers for an electro-hydraulic position servo system," Int. J. 659 Adv. Manuf. Technol., vol. 26, no. 1, pp. 117-123, 2005.

12] A. Ghazy "Variable structure control for electrohydraulic position 661 servo system," in Proc. IEEE 27th Annu. Conf. Ind. Electron. Soc., 2001, 662 pp. 2194-2198.

[13] C. Guan and S. Pan, "Adaptive sliding mode control of electro-hydraulic 664 system with nonlinear unknown parameters," Control Eng. Pract., 665 vol. 16, no. 11, pp. 1275-1284, Nov. 2008.

[14] Y. Lin, Y. Shi, and R. Burton, "Modeling and robust discrete-time sliding 667 mode control design for a fluid power electro-hydraulic actuator (EHA) 668 system," IEEE/ASME Trans. Mechatronics, vol. 18, no. 1, pp. 1-10, Feb. 669 2013

[15] A. G. Loukianov, J. Rivera, Y. Orlov, and E. Teraoka, "Robust trajectory tracking for an electrohydraulic actuator," IEEE Trans. Ind. Electron., vol. 56, no. 9, pp. 3523-3531, Sep. 2009.

[16] B. Yao, F. Bu, J. Reedy, and G. T. C. Chiu, "Adaptive robust motion 674 control of single-rod hydraulic actuators: Theory and experiments," 675 IEEE/ASME Trans. Mechatronics, vol. 5, no. 1, pp. 79-91, Mar. 2000.

[17] B. Yao, F. Bu, and G. T. C. Chiu, "Nonlinear adaptive robust con- 677 trol of electro-hydraulic systems driven by double-rod actuators," Int. J. 678 Control., vol. 74, no. 8, pp. 761-775, Aug. 2001. 
[18] G. Cheng and P. Shuangxia, "Nonlinear adaptive robust control of single-rod electro-hydraulic actuator with unknown nonlinear parameters," IEEE Trans. Control Syst. Technol., vol. 16, no. 3, pp. 434-445, May 2008.

[19] C. Wang, Z. Jiao, S. Wu, and Y. Shang, "Nonlinear adaptive torque control of electro-hydraulic load system with external active motion disturbance," Mechatronics, vol. 24, no. 1, pp. 32-40, Feb. 2014.

[20] Y. Cungui and Q. Xianwei, "Simplified adaptive robust motion control with varying boundary discontinuous projection of hydraulic actuator," Math. Prob. Eng., vol. 2014.

[21] A. Alleyne and R. Liu, "A simplified approach to force control for electrohydraulic systems," Control Eng. Pract., vol. 8, no. 12, pp. 1347-1356. Dec. 2000.

[22] C. Kaddissi, J.-P. Kenne, and M. Saad, "Identification and real-time control of an electrohydraulic servo system based on nonlinear backstepping," IEEE/ASME Trans. Mechatronics, vol. 12, no. 1, pp. 12-22, Feb. 2007.

[23] C. Kaddissi, J.-P. Kenne, and M. Saad, "Indirect adaptive control of an electrohydraulic servo system based on nonlinear backstepping," IEEE/ASME Trans. Mechatronics, vol. 16, no. 6, pp. 1171-1177, Dec. 2011.

[24] K. K. Ahn, D. N. C. Nam, and M. Jin, "Adaptive backstepping control of an electrohydraulic actuator," IEEE/ASME Trans. Mechatronics, vol. 19, no. 3, pp. 987-995, Jun. 2014.

[25] P. Nakkarat and S. Kuntanapreeda, "Observer-based backstepping force control of an electrohydraulic actuator," Control Eng. Pract., vol. 17, no. 8, pp. 895-902, 2009.

[26] K. Wonhee, W. Daehee, S. Donghoon, and C. Chung, "Output feedback nonlinear control for electro-hydraulic systems," Mechatronics, vol. 22, no. 6, pp. 766-777, Sep. 2012.

[27] X. Wang, X. Sun, S. Li, and H. Ye, "Output feedback domination approach for finite-time force control of an electrohydraulic," IET Control Theory, vol. 6, no. 7, pp. 921-934, 2011.

[28] H. Khan, S. C. Abou, and N. Sepehri, "Nonlinear observer-based fault detection technique for electro-hydraulic servo-positioning systems," Mechatronics, vol. 15, no. 9, pp. 1037-1059, 2005.

[29] K. Wonhee, W. Daehee, S. Donghoon, and C. Chung, "Disturbanceobserver-based position tracking controller in the presence of biased sinusoidal disturbance for electrohydraulic actuators," IEEE Trans. Control Syst. Technol., vol. 21, no. 6, pp. 2290-2298, Nov. 2013.

[30] C. S. Kim and C. O. Lee, "Speed control of an overcentered variable displacement hydraulic motor with a load torque observer," Control Eng. Pract., vol. 4, no. 11, pp. 1563-1570, 1996.

[31] W. Daehee, K. Wonhee, S. Donghoon, and C. Chung, "High-gain disturbance observer-based backstepping control with output tracking error constraint for electro-hydraulic systems," IEEE Trans. Control Syst. Technol., vol. 23, no. 6, pp. 758-795, Mar. 2014.

[32] J. Yao, Z. Jiao, and D. Ma, "Extended-state-observer-based output feedback nonlinear robust control of hydraulic systems with backstepping," IEEE Trans. Ind. Electron., vol. 61, no. 11, pp. 6285-6293, Nov. 2014.

[33] J. Yao, Z. Jiao, and D. Ma, "Adaptive robust control of dc motors with extended state observer," IEEE Trans. Ind. Electron., vol. 61, no. 7, pp. 3630-3637, Jul. 2014.

[34] S. Ahmed Ali, "Sampled data observer based inter-sample output predictor for electro-hydraulic actuators," ISA Trans., vol. 58, pp. 421-433, 2015.

[35] I. Karafyllis and C. Kravaris, "From continuous-time design to sampleddata design of observers," IEEE Trans. Autom. Control, vol. 54, no. 9, pp. 2169-2174, Sep. 2009.

[36] A. Karakayis and S. Begg, "Investigation of control system strategies for hydraulic valve actuation in an IC engine Adil Karakayis," M.S. thesis, Dept. Autom. Eng., Univ. Brighton School Comput., Eng. Math., Div. Eng. Product Des., Brighton, U.K., 2014.

[37] W. Sun, Z. Zhao, and H. Gao, "Saturated adaptive robust control for active suspension systems," IEEE Trans. Ind. Electron., vol. 60, no. 9, pp. 38893896, Sep. 2013.

[38] W. Sun, H. Gao, and B. Yao, "Adaptive robust vibration control of full-car active suspensions with electrohydraulic actuators," IEEE Trans. Control Syst. Technol., vol. 21, no. 6, pp. 2417-2422, Nov. 2013.

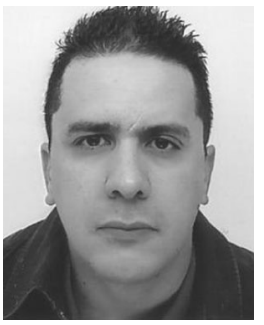

Sofiane Ahmed Ali was born in Algiers, 749 Algeria, in 1977. He received the B.Sc. degree 750 in electrical engineering from the University 751 of Technology Houari Boumediene, Algiers, 752 Algeria, in 2001, and the M.Sc. and Ph.D. 753 degrees in electrical and computer engineer- 754 ing from the University of Le Havre, Le Havre, 755 France, in 2004 and 2008, respectively. $\quad 756$

In 2008, he was appointed as a Research 757 and Development Engineer with Renault. Since 758 2010, he has been a Teaching and Research 759 Assistant Professor with the École Supérieure d'Ingénieurs en Génie 760 Électrique (ESIGELEC), Rouen, France. His research interests include 761 sliding mode control, nonlinear observers and fault-tolerant control, and 762 diagnosis in the field of mechatronics devices.

763Q5

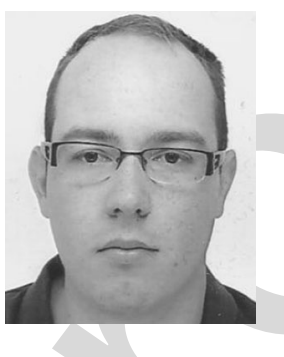

Arnaud Christen was born in France, in 764 1991. He received the Baccalaureate degree 765 in science (with honors) in 2009, before fol- 766 lowing a two-year preparation in mathemat- 767 ics and physics for entrance to the French 768 Engineering Schools. He received the dual M.S. 769 degree in control theory (electrical engineer- 770 ing) and mechatronics from École Supérieure 771 d'Ingénieurs en Génie Électrique (ESIGELEC), 772 Rouen, France, and the University of Rouen, 773 Rouen, France, in 2015.

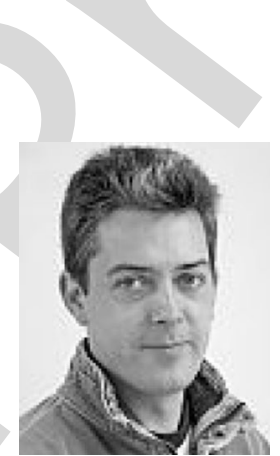

Steven Begg received the B.Eng. degree (with 775 honors) in mechanical engineering and the Ph.D. $776 \mathrm{Q} 7$ degree from the University of Brighton, Brighton, 777 U.K., in 2003.

$\mathrm{He}$ is a Reader, Fellow of the Higher 779 Education Academy, and the Course Leader 780 for the Automotive Engineering undergraduate 781 degree pathways in the School of Computing, 782 Engineering and Mathematics, University of 783 Brighton. He is the Leader of the Experimental 784 Fluid Mechanics Research Group and a Member 785 of the Advanced Engineering Centre at Brighton, U.K. He has led 786 applied research programmes (EPSRC, DfT, DTI, TSB, and EU) as 787 well as industrial consultancy projects, in the fields of automotive engi- 788 neering, fluid mechanics, and optical diagnostic techniques, for over 21789 years.

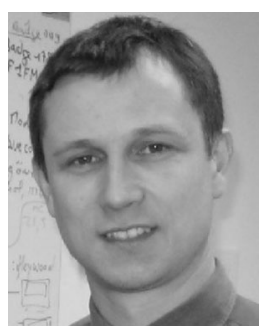

Nicolas Langlois received the Ph.D. and HDR 791 (habilitation to supervise research) degrees in 792 automatic control and signal processing from the 793 University of Rouen, Rouen, France, in 2001 and 794 2008, respectively.

In 2000, he joined the Graduate School 796 of Electrical Engineering, ESIGELEC, Rouen, 797 France. $\mathrm{He}$ is currently the Head-in-Charge 798 of skills acquisition through research of 799 ESIGELEC, where he teaches courses on 800 control systems and digital signal processing. 801 $\mathrm{He}$ has also the Head of the "Automatic Control and Systems" research 802 team at the research institute IRSEEM since 2008. His research 803 interests include fault-tolerant control. 


\section{QUERIES}

Q1: Please provide expansion for "PI."

Q2: Please provide expansion for "IRSEEM."

Q3: As per IEEE style, vectors have been changed to boldface italic. Please check whether all the occurrences are identified correctly and specify the missed out occurrences.

Q4: Please provide complete details of Refs. [9] and [20].

Q5: Please provide the location for Renault in the biography section of the author Sofiane Ahmed Ali.

Q6: Please provide the institution name and location for the Baccalaureate degree of the author Arnaud Christen.

Q7: Please spell out the term "ESIGELEC."

Q8: Please provide field of study for the Ph.D. degree of author "Steven Begg."

Q9: Please expand "IRSEEM" in the biography section. 